\title{
Laplace pressure evolution and four instabilities in evaporating two-grain liquid bridges
}

\author{
Boleslaw Mielniczuk a,b,d,*, Tomasz Hueckel ${ }^{c}$, Moulay Saïd El Youssoufi d,b \\ a Institut de Radioprotection et de Sûreté Nucléaire, IRSN, B.P. 3, 13115 Saint-Paul-lez-Durance Cedex, France \\ b Laboratoire de Micromécanique et d'Intégrité des Structures MIST, IRSN-CNRS-Université de Montpellier, France \\ c Duke University, Durham, NC 27708, USA \\ d Laboratoire de Mécanique et Génie Civil UMR UM-CNRS 5508, Université de Montpellier, 34095 Montpellier Cedex 5, France
}

\section{A R T I C L E I N F O}

\section{Article history:}

Received 4 March 2015

Received in revised form 4 May 2015

Accepted 17 May 2015

Available online 23 May 2015

\section{Keywords:}

Evaporation

Capillary bridge

Intergranular forces

Laplace pressure

Contact line

\begin{abstract}
A B S T R A C T
Dynamic variables characterizing evolution during evaporation of capillary bridge between two spheres are analyzed. The variables include: average Laplace pressure, pressure resulting force, surface tension force and total capillary force calculated based on the previously reported geometrical variables using Young-Laplace law $[1,2]$. This is the first time to our knowledge that Laplace pressure is calculated from the measured bridge curvatures along the process of evaporation and compared to experimental measurement data. A comparison with the experimental data from analogous capillary bridge extension tests is also shown and discussed.

The behavior of evaporating liquid bridges is seen as strongly dependent on the grain separation. Initial negative Laplace pressure at small separations is seen to significantly augment during an advanced stage of evaporation, but to turn into positive pressure, after an instability toward the end of the process, and prior to rupture. At larger separations the pressure is positive all the time, changing a little, but rupturing early. Rupture in all cases occurs at positive pressure. However, because of the evolution of the surface area of contact, the resultant total capillary forces are always tensile, and decreasing toward zero in all cases. Comparison between measured total resultant capillary forces and those calculated from the Young-Laplace law is very good, except for some discrepancies at very small separations (below $50 \mu \mathrm{m}$ ). Up to four consecutive instabilities of capillary bridge are seen developing at some sphere separations. They are: re-pinning-induced suction (pressure) instability; Rayleigh nodoid/ catenoid/unduloid unstable transition, associated with zero-pressure; Rayleigh unduloid/cylinder unstable transition, associated with the formation of a liquid-wire; and lastly, a pinching instability of the liquid-wire, associated with the bridge rupture. Rupture of the bridges is seen at large separations to occur quite early, at only $1 / 4-1 / 3$ of the initial water volume evaporated. At smallest separations, rupture occurs in a seemingly unstable way when water evaporates from the bridge thinnest section of the neck.
\end{abstract}

(c) 2015 Elsevier B.V. All rights reserved.

\section{Introduction}

Evaporating capillary bridges between two glass spheres at fixed separations revealed in recent isothermal experiments characteristic patterns of their evolution during liquid evaporation [1]. That includes total mass-evaporation rate, average evaporation flux, evolution of geometric characteristics, such as the radius of bridge gorge, central external radius, angles and radii of contact, and evolution of capillary force. These patterns bear similarities to, as well as differences from, the analogous quantities measured during more familiar short-term axial extension tests of such bridges, with negligible evaporation. An important part of such evolution is rupture of liquid bridges at some

\footnotetext{
* Corresponding author.

E-mail addresses: boleslaw.mielniczuk@univ-montp2.fr (B. Mielniczuk), hueckel@duke.edu (T. Hueckel), Moulay-Said.El-Youssoufi@univ-montp2.fr (M.S. El Youssoufi).
}

separations and other forms of bridge instability [2]. An importance of a better understanding of rupture conditions comes from the role of liquid bridges in mechanical strengthening of granular media, and in the change of liquid repartition pattern.

The objective of this paper is to process, analyze, and discuss the numerical data from the aforementioned experiments on the evaporationinduced evolution of the following bridge characteristics: gorge radius, $r_{g}$ and of a mean value of two measured external radii, $r_{\text {ext }}$ (Fig. 1) for a series of separations between grains $D$, to calculate the Laplace pressure, $\Delta p$ and Laplace pressure resulting force, $\mathrm{F}_{\Delta \mathrm{p}}$ as well as the surface tension component of the evolving capillary force $\mathrm{F}_{\mathrm{ST}}$ with the use of "gorge method" [3]. The use of the gorge and a single external radius is equivalent to treating the bridge as a structured water body of a uniform external radius of curvature, and implying a uniform liquid pressure throughout.

The evolution of contact angle (hysteresis), pinning, de-pinning and re-pinning of the contact line are reported by Mielniczuk et al. [1], but are 


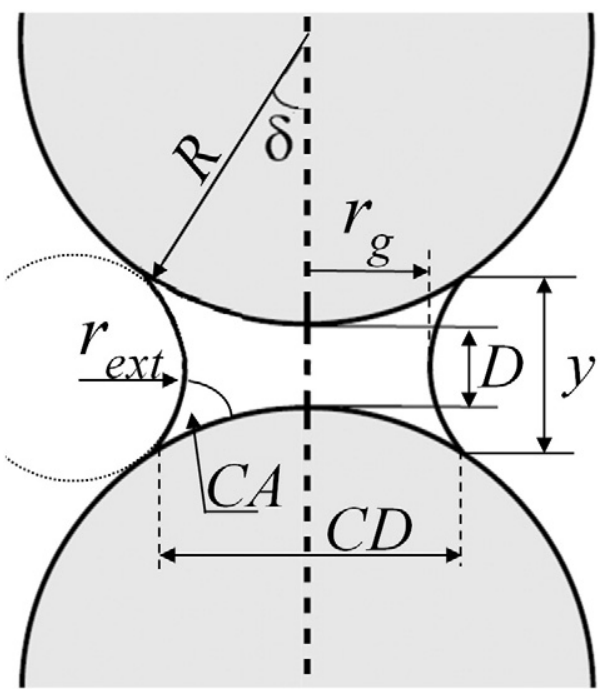

Fig. 1. Liquid bridge between two spheres.

not considered in Laplace-Young law and in the expression for the Laplace pressure. However, as seen, they heavily affect the evolution of Laplace pressure. The calculated total capillary force $F_{C A P}$ being a resultant of the pressure force $F_{\Delta \mathrm{p}}$, and the surface tension components $F_{S T}$, is compared to the values obtained experimentally. A similar comparison is also made with, and discussed in the context of the analogous conditions during capillary bridge extension (separation increase) tests. Rigid spheres of equal radius and constant separation systems are considered only.

\section{Experimental input}

The main dynamic data from our recent experiments (see [1] for details) are synthetized in Fig. 2a and b, visualizing the total measured capillary force, $F_{C A P-m}$ against the current volume of the liquid bridge and separation between the spheres. A description of the experimental conditions is provided by [1].

The curves at constant separations $D$ from 0.01 to $2.0 \mathrm{~mm}$ were obtained in evaporation driven tests, all starting from the same initial liquid volume of $4.0 \mu$ l. The curves at constant volume $V$ were obtained from extension tests, all started at a zero separation.

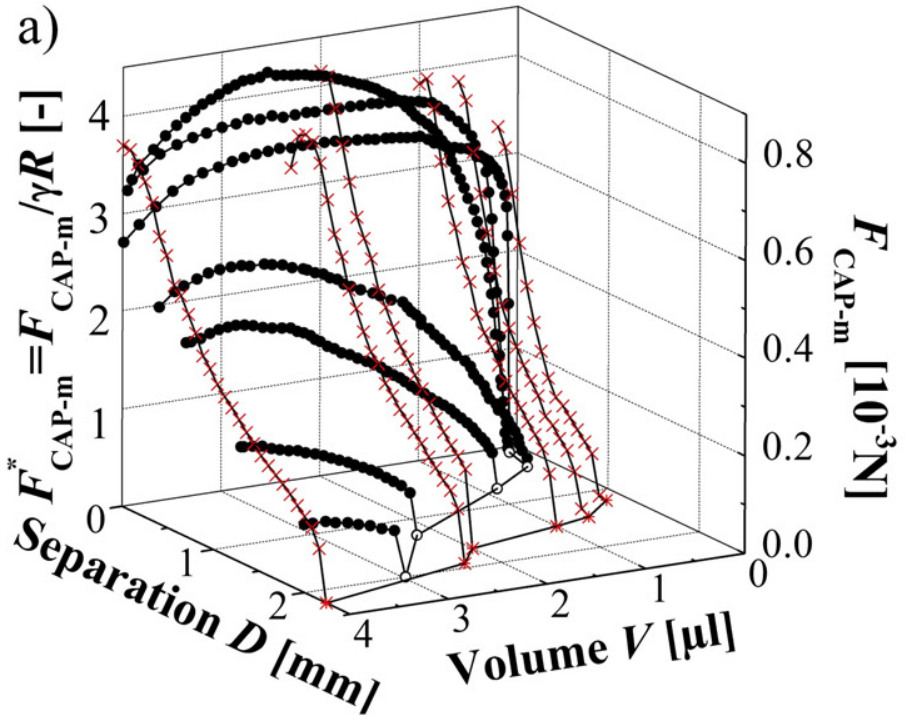

All the data of total capillary force denoted in what follows as "measured", both during evaporation and extension, in reality were calculated from the truly measured total intergranular force by subtracting from the latter the hydrostatic pressure resultant force (weight effect), acting on the surface area of cross-section at gorge level, as proposed by Princen [4] and Adams et al. [5]. This was performed to be able to directly compare the force $F_{C A P-m}$ to the one calculated from the curvature radii, $F_{C A P}$. The gravity (hydrostatic) force contribution has nevertheless been very small, never higher than $5 \%$ of the total measured intergranular force.

In general, the capillary force, $F_{C A P-m}$ (Fig. 2) decreases, both during evaporation and during extension. There is a difference in this trend for the two processes: the capillary force is a convex function (of liquid volume loss) for evaporation (slow evolution at the beginning, fast at the end), while for extension the capillary force is largely a concave function of varying separation at constant volume (fast evolution at the beginning and slow at the end).

Both graphs indicate that the surface $F_{C A P-m}(V, D)$ hypothetically spanning the constant separation (evaporation) curves does not exist in a relatively vast range of liquid volumes (less than $2 \mu \mathrm{l}$, at $D=1.3 \mathrm{~mm}$, and less than $1 \mu \mathrm{l}$, at $D=0.7 \mathrm{~mm}$ ) as marked on the "floor" of the graph, while it does certainly exist in that range for the extension processes. The principal cause of that is rupture of the bridges occurring within the domain and hence cutting off portions of the range. Apart from the rupture range, there are differences between the capillary force value for evaporation and extension that may reach up to 80 $\%$ of the maximum value (e.g. for separation of $0.1 \mathrm{~mm}$ ). The overall conclusion from these observations is that the capillary force, $F_{C A P-m}$ is not a state function of volume and separation [1], but does depend on the history of evaporation or extension and on the solid-liquid contact evolution, in particular the history of contact diameter $C D$ and contact angle $C A$.

\subsection{Radii of curvature of liquid bridge and their evolution}

To quantify the evolution of the profile of the liquid body, the two principal geometric characteristics of the liquid bridges were determined by image-processing: the radii of their gorge $r_{g}$ and of the external meridian curvature $r_{\text {ext }}$ (and hence, the mean surface curvature). These two variables enter the Laplace-Young law for capillary pressure, which is a macroscopic equilibrium theory based on the assumption of constant pressure throughout the bridge.

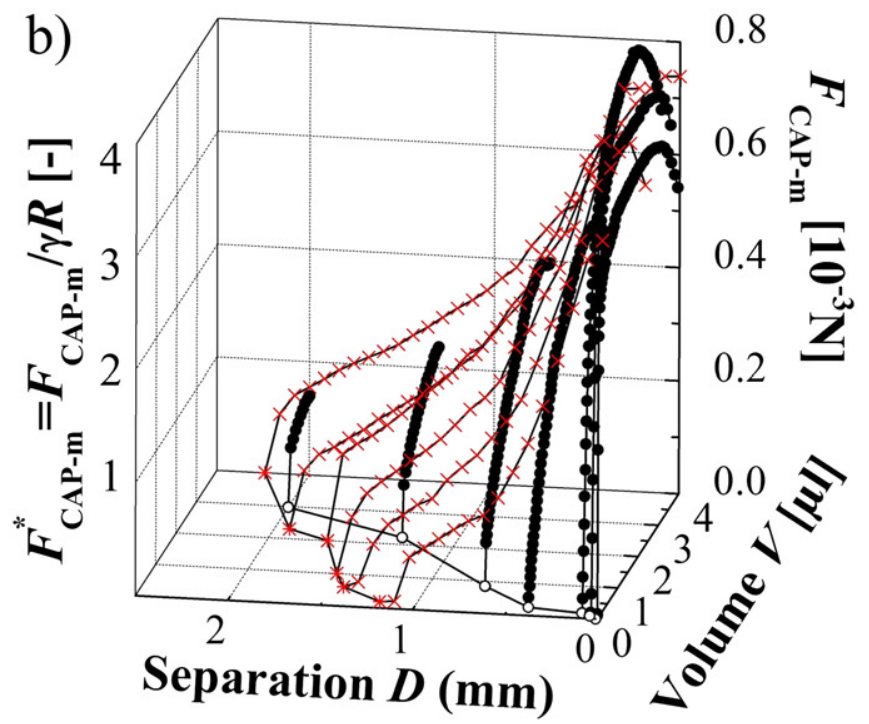

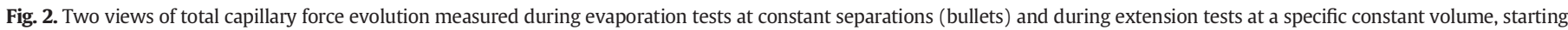

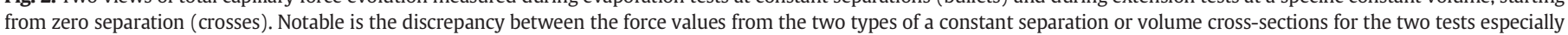
marked at smaller liquid volumes and smaller separations. 
The results are summarized in Fig. 3a and b, showing the gorge radius $r_{g}$ and external radius $r_{\text {ext }}$ evolution versus the current volume $V$ of the liquid bridge, (bullets) and the current separation $D$ between the spheres (crosses). The data of the radius evolutions were obtained with a still camera, Canon EOS500 with a macro lens. Images were saved each minute for the evaporation tests and each $5 \mathrm{~s}$ for the extension tests, with a resolution of 12 megapixels.

\subsection{Gorge radius}

During evaporation the initial $r_{\mathrm{g}}$ varies between $1.71 \mathrm{~mm}$ at separation $D=0.01 \mathrm{~mm}$ and $0.62 \mathrm{~mm}$ at $D=2 \mathrm{~mm}$, with much thinner bridges for higher separations (obviously, as the initial volume is the same by choice). During evaporation, the initially linear function $r_{g}(V)$ decreases and it looses the linearity after more than $2 / 3$ through the process. For larger separations, the bridge evolution terminates early with a bridge rupture (at $1 / 4$ to $3 / 4$ of water evaporated), with the critical (final) $r_{g}$ between 0.375 and $0.01 \mathrm{~mm}$ (Fig. 4a). For the smaller separations $(D<0.7 \mathrm{~mm})$ a rapid, visibly unstable decrease of $r_{g}$ occurs near the end of the process.

Set up for extension tests the initial $r_{g}$ vary between $1 \mathrm{~mm}$ at $V=$ $0.2 \mu \mathrm{l}$ and $2.27 \mathrm{~mm}$ at $V=10 \mu \mathrm{l}$ (Fig. $4 \mathrm{~b}$ ). A linear decrease of $r_{g}(D)$ during the extension has a consistently lower rate of decrease at the terminal separations. The final gorge radius is from $0.243 \mathrm{~mm}$ at $0.2 \mu \mathrm{l}$ to $0.579 \mathrm{~mm}$ at $V=10 \mu \mathrm{l}$ (Fig. 4b).

It is important to note a difference in the rate of decrease of gorge radius, which largely controls both: $F_{\Delta p}$ and $F_{S T}$, as seen in what follows. While for extension the rate of $r_{g}$ decrease is nearly a linear function of separation, for evaporation a clear acceleration toward the end of the process is visible, especially for smaller values of $D$.

\subsection{External curvature radius}

The external radius $r_{\text {ext }}$ decreases monotonically during evaporation in a similar way for all the separations (Fig. 3b, bullets). The initial values are fairly similar for all the separations, whereas the terminal values range from nearly zero at small separations, to relatively high (about $50 \%$ of the original values) at the highest separations (Fig. $4 \mathrm{a}$ ).

Evolution of $r_{\text {ext }}$ during extension is relatively modest. Its initial and final values are almost the same for all volumes (Fig. 4b). For each liquid volume the curve $r_{\text {ext }}(D)$ has two extrema: with an increasing separation it initially decreases, reaches a minimum, then it increases to a maximum and decreases again till rupture (Fig. 3b, crosses). This is consistent for all water volumes tested, except for the smallest (at $V=0.2 \mu \mathrm{l}$ ), for which the first minimum coincides with the beginning of the process. As for the dependence on the liquid volume $r_{e x t}(V)$, the

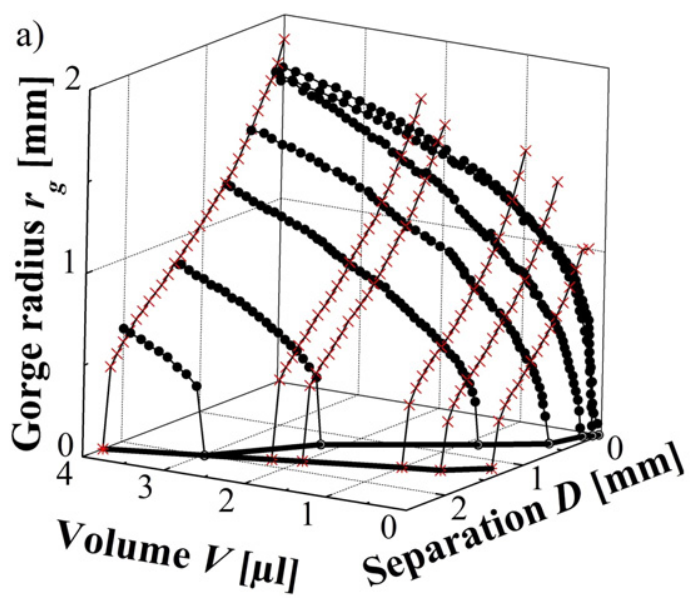

initial and final radii both increase, with the exception of the final $r_{e x t}$ that has a noticeable maximum at $4 \mu \mathrm{l}$ (Fig. $4 \mathrm{~b}$ ).

\section{Evolution of calculated Laplace pressure (suction), resultant pressure force, surface tension force and total capillary force}

The force of capillary interaction between the grains has been originally attributed entirely to the gas and liquid pressure difference resultant [6]. Fisher [7] has convincingly argued and proved that the perimeter surface tension resultant plays an equally important role in the total force of inter-grain interaction. Therefore, the total capillary force, $F_{C A P}$ (positive when attractive) is now believed to be a sum of the force of liquid pressure difference resultant $F_{\Delta p}$ and the resultant surface tension force $F_{S T}$ (vertical component of the surface tension acting at the perimeter of the gorge), The gravity force is considered here as negligible $[8,9]$, hence

$F_{C A P}=F_{\Delta p}+F_{S T}$

The pressure resultant force between two wet grains is [6,10]

$F_{\Delta p}=-\pi r_{g}^{2} \Delta p$

where $\Delta p$ is the difference of external (ambient gas) and internal (inside bridge) liquid pressure (Laplace pressure), resulting from YoungLaplace law as proportional to the mean surface curvature at the symmetry plane meeting the point

$\Delta p=\gamma\left(\kappa_{g}+\kappa_{\text {ext }}\right)$

where the curvatures $\kappa_{g}=r_{g}{ }^{-1}$ and $\kappa_{e x t}=r_{e x t}{ }^{-1}$ are considered positive for convex curves and negative for the concave ones; $\gamma$ is surface tension of the liquid determined experimentally.

The surface tension force acting on the bridge perimeter at its neck is $[7,9,11]$

$F_{S T}=2 \pi r_{g} \gamma$

The liquid is a mixture being used at the University of Montpellier for experiments on capillary forces, see e.g. [12]. The mixture composes of the milli-Q ultrapure water and some ingredients acquired by water when it is circulated through the hydraulic system of the apparatus (not relevant in our experiments). The surface tension coefficient $\gamma$ of the liquid used in further analyses is determined experimentally with use of Wilhelmy plate method [13], with assumption of zero contact angle. The experiment was repeated several times and the mean value was determined as $\gamma_{s}=0.0496 \mathrm{~N} / \mathrm{m}$. Similar values were reported by Gras (mean value of $\gamma_{s}=0.0510 \mathrm{~N} / \mathrm{m}$, [12]). These values are notably

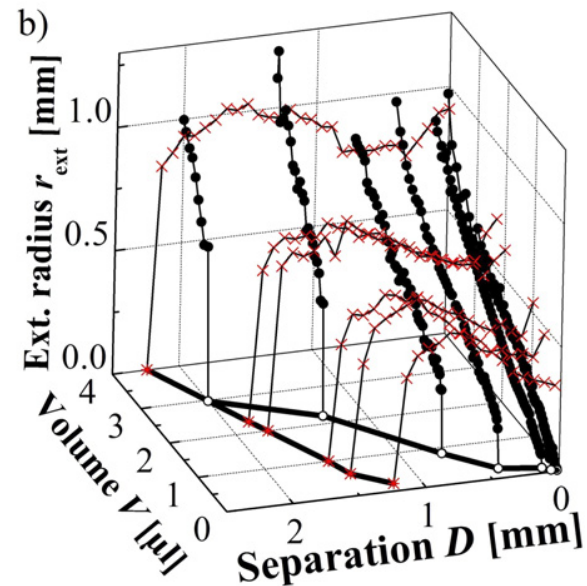

Fig. 3. Liquid gorge radii evolution in evaporation tests at constant separations, or in extension tests at constant volumes of the liquid bridge: a) gorge radius; b) external radius. 

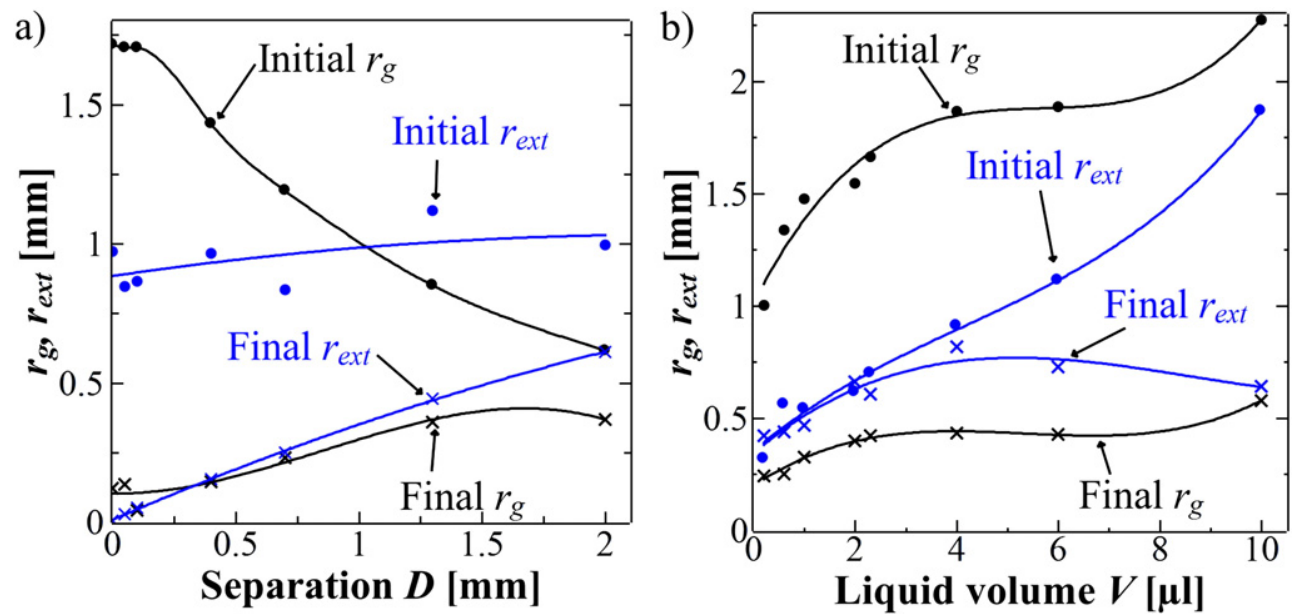

Fig. 4. Initial and terminal radii: (a) for different evaporation tests at $4 \mu \mathrm{l}$ (initial) with different separations, (b) for different extension tests with different liquid volumes.

lower than the nominal value of $0.0728 \mathrm{~N} / \mathrm{m}$ for the surface tension of distilled water. Surface tension is notoriously sensitive to the presence of contaminants (see, e.g. $[14,15]$ ). All the force related results are anyway normalized with respect to surface tension.

By Young-Laplace law, the dynamics of the liquid bridge body is entirely controlled by its geometry, in particular, its two principal curvatures $\kappa_{\mathrm{g}}$ and $\kappa_{\text {ext }}$. Based on the previous results [1], we assess the role of the two principal components (suction and surface tension) in the evolution of the capillary force during the process of evaporation.

\subsection{Laplace pressure}

The variation of $\Delta p$ calculated following the Eq. (3) is shown in Fig. 5. A possible influence of a liquid film extending beyond the area of the actual bridge is neglected, as no such film has been detected.

The results reveal a relatively similar trend in the evolution of $\Delta p$ during both evaporation and extension, but with a few important exceptions.

In general, liquid in a capillary bridge initially develops a negative $\Delta p$, from about $-5 \mathrm{~Pa}$ at $V=10 \mu \mathrm{l}$ up to $-106 \mathrm{~Pa}$ at $V=0.2 \mu$ l. Only at the highest separations $D$ at evaporation tests $(1.3$ and $2.0 \mathrm{~mm})$ the initial $\Delta p$ is positive, and stays that way during the entire process of evaporation up to rupture (about 53 Pa for $D=2 \mathrm{~mm}$ ).

For evaporation, for all separations between $0.1 \mathrm{~mm}$ and $0.7 \mathrm{~mm}$ the pattern of evolution of $\Delta p$ is similar. The initial liquid bridge is under suction, which gradually increases during evaporation, to a maximum that is up to three times the initial value (e.g. $\Delta p=102$ Pa for $D=$ $0.4 \mathrm{~mm}$ ) at a $1 / 3 \mathrm{rd}$ to $1 / 4$ th of the water volume left). Shortly after the maximum a sharp decrease of suction to zero is visible and positive pressure develops with values reaching about $25 \mathrm{~Pa}$ for $D=0.4 \mathrm{~mm}$ and up to 196 Pa for $D=0.1 \mathrm{~mm}$, before the rupture of the bridge (at the 1 min time resolution). For the smallest separations $(D<0.1)$ a maximum suction may achieve values as high as $10 \mathrm{kPa}$ just before the rupture, with about $10 \%$ of the initial water left (see inset in Fig. 5a)

However, when the images are processed of the last few milliseconds, prior to rupture, as recorded with the ultra-fast camera (see [2]), the pattern of Laplace pressure evolution acquires additional a notable ending, see Fig. 6. The positive $\Delta p$ that develops in the terminal phase of evaporation reaches two-order of magnitude higher values, up to $2 \mathrm{kPa}$, than those calculated from the slow camera photos. That
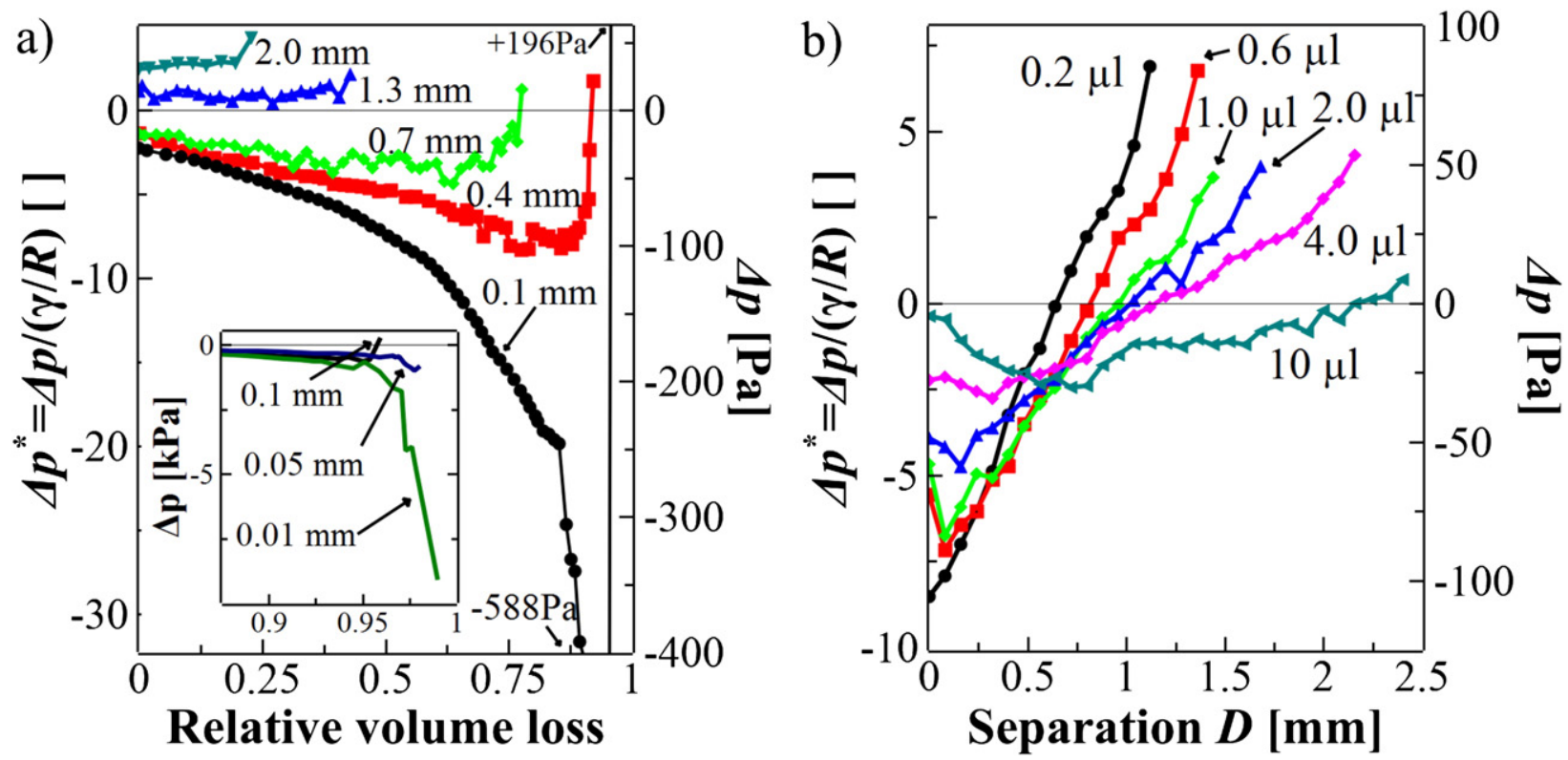

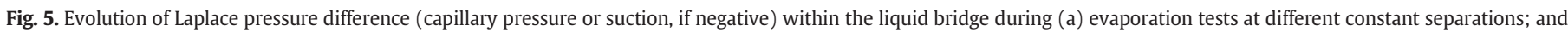
(b) extension tests at different constant liquid volumes. The values are calculated from the measured radius values following Laplace equation. 

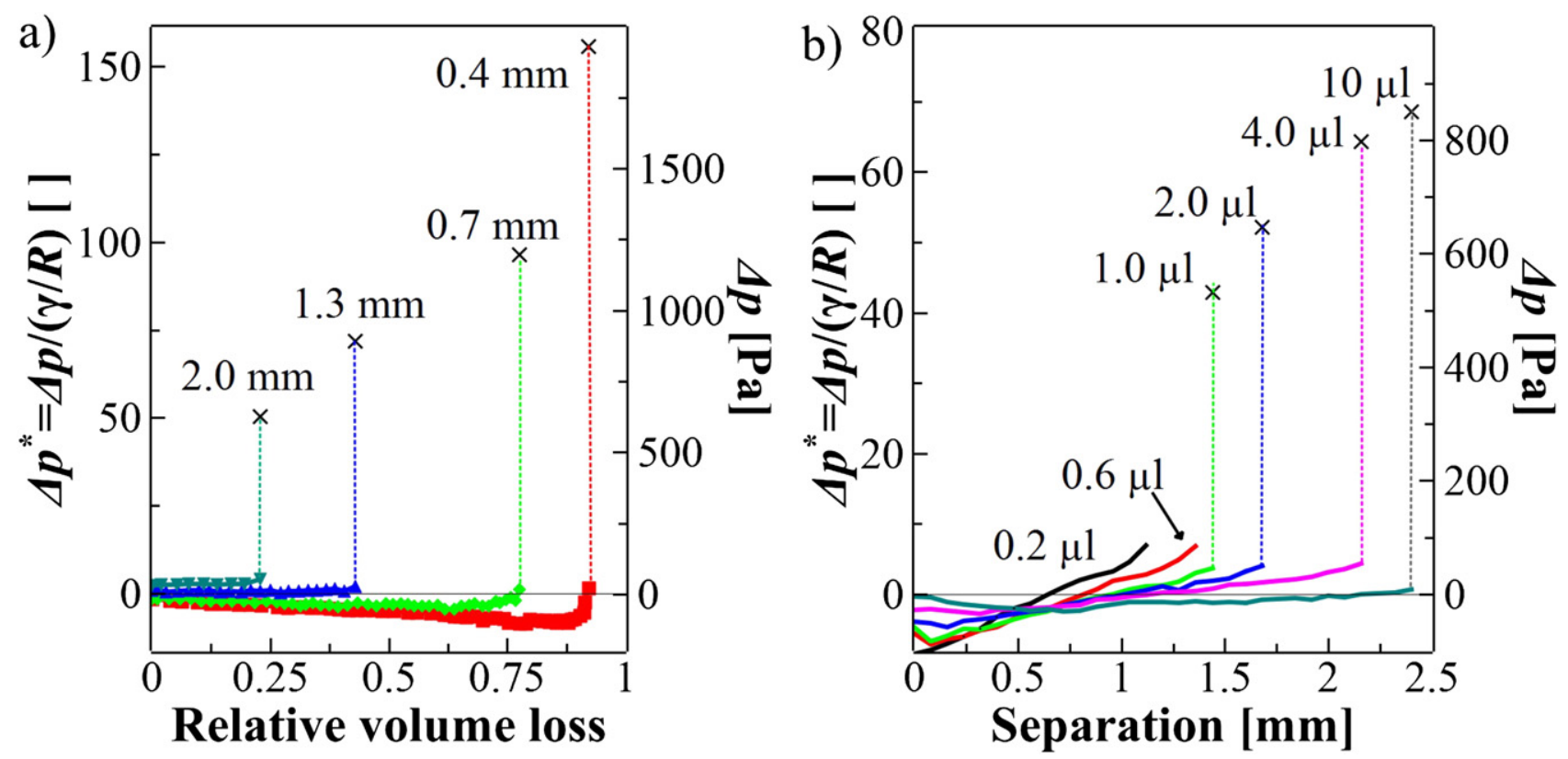

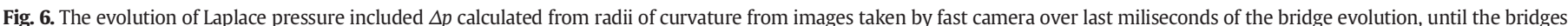

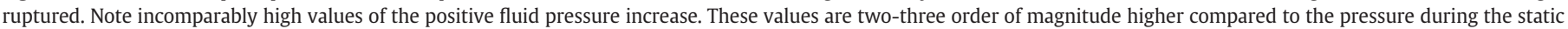
evolution.

corresponds to the phase when the bridge evolves into a water-wire, with a very high and increasing gorge curvature and almost zero external curvature over almost entire length of the wire. In that situation, $\Delta p$ increases dramatically, until interrupted by an instability of the waterwire rupture (see [2]).

For extension, the initial phase of $\Delta p(D)$ evolution (Fig. 5b) is characterized by a brief stage of a modest suction increase to a maximum: the smaller the liquid volume, the higher and earlier the maximum suction. At the maximum the curve is much sharper than in the case of evaporation test. After the maximum, there is a prolonged phase of a nearly linear suction decrease continuing into a significant phase of positive pressure in the liquid body, with the final $\Delta p$ visibly higher than those at the end of evaporation. Interestingly, for the highest liquid volume, the initial and terminal $\Delta p$ is about zero. The terminal pulse of positive pressure as seen with fast camera is also visible in extension tests, associated with the water-wire effect, however reaching somewhat smaller final values (see Fig. 6b).

There are a few particular conclusions from the above assessment of the Laplace pressure. In both cases, the pressure evolution scenario is similar: an initial increase of suction, followed by its extensive decline and then a gradual process of pressurization of the liquid bridge, with the rupture in the presence of a positive $\Delta p$, which is quite counterintuitive. For extension, the behavior of water body resembles behavior of extended elastic solid bar, which is under negative mean stress. However, as opposed to the extension of elastic solids, the capillary force decreases after a maximum, like in solids with plastic softening or being damaged [16,17].

The expectation for a negative $\Delta p$ in the case of evaporation comes from a vast macroscopic scale experimental evidence of an increasing suction during drying of granular media reaching elevated values reportedly as high as $2.0 \mathrm{MPa}$ [18] or more than $100 \mathrm{MPa}$ [19-21] at the degree of saturation below 5\%. This is still true in our experiments at the micro-scale in the initial phase of evaporation, but not anymore in the more advanced one, and near rupture. It is to be remembered that what constitutes an early stage of loss of water from the capillary bridge, on the macro-scale corresponds to a very low saturation degree, (about $9 \%$ for a cubic packing systems, see [22]). However, it needs to be stressed that most of the macroscopic desaturation tests are suction controlled. In our tests, suction arises in response to the evaporation flux, and is calculated from the geometric data, assuming the validity of Young-Laplace law. In all our attempts to directly measure $\Delta p$ at the micro-scale, the measurement resulted to be a highly invasive process.

\subsection{Intergranular resultant forces of pressure and surface tension}

From the point of view of a number of technologies, a variable of importance is a capillary force, rather than Laplace pressure $\Delta p$. The total capillary force $F_{C A P}$ (usually computed at the bridge gorge) is calculated as a sum of the two components (Eq. (1)) corresponding to $\Delta p$ acting over the bridge cross section surface area $\left(F_{\Delta \mathrm{p}}\right.$, Eq. $\left.(2)\right)$ and surface tension acting over the length of the perimeter $\left(F_{S T}\right.$, Eq. $(4)$ ), following the original experimental proof by Fisher [7].

The variation of $\Delta p$ is therefore altered by a quadratic function of an intensely evolving $r_{g}$, as shown in Fig. 3 . On the other hand, the surface tension force $F_{S T}$ is a linear function of the same variable $r_{g}$. The computed resultant forces $F_{\Delta p}$ and $F_{S T}$ for evaporation and extension are presented in Figs. 7 and 8.

Laplace pressure resulting force $F_{\Delta \mathrm{p}}$ for evaporation is generally positive (attractive) except for the largest separations ( $D=1.3$ and $2 \mathrm{~mm}$ ) and just before the rupture, when it results in a slightly repulsive force. The values of the initial negative $\Delta p$ (Fig. 5a) became magnified by the cross section surface area of the gorge at the beginning of the process, but the positive pressure contribution toward the end of the process is substantially diminished, as the gorge area becomes very small. As a result, the positive pressures developing in the liquid toward the end of evaporation turn out to minimally contribute to $F_{\Delta p}$. Nevertheless, during evaporation $F_{\Delta \mathrm{p}}$ 's value is doubled at the maximum, to eventually rapidly decline until reaching practically zero in all cases. For the largest separations $F_{\Delta p}$ originates and remains slightly repulsive until it vanishes with the vanishing volume of water (Figs. 7a, 9a).

The surface tension force $F_{S T}$ during evaporation mirrors the evolution of the gorge radius (Fig. 3a) as expected from Eq. (4). For all examined separations, it is hence positive and it decays as a convex (for evaporation) or concave (for extension) parabola. Initial value of $F_{S T}$ is the highest for the lowest $D$, and it decreases with the increasing $D$, whereas the final values have an opposite trend: zero for the smallest $D$ and the highest for large $D$ (Fig. 7b). Numerically, both forces $F_{\Delta \mathrm{p}}$ and $F_{\mathrm{ST}}$ are of a similar order. 
a)

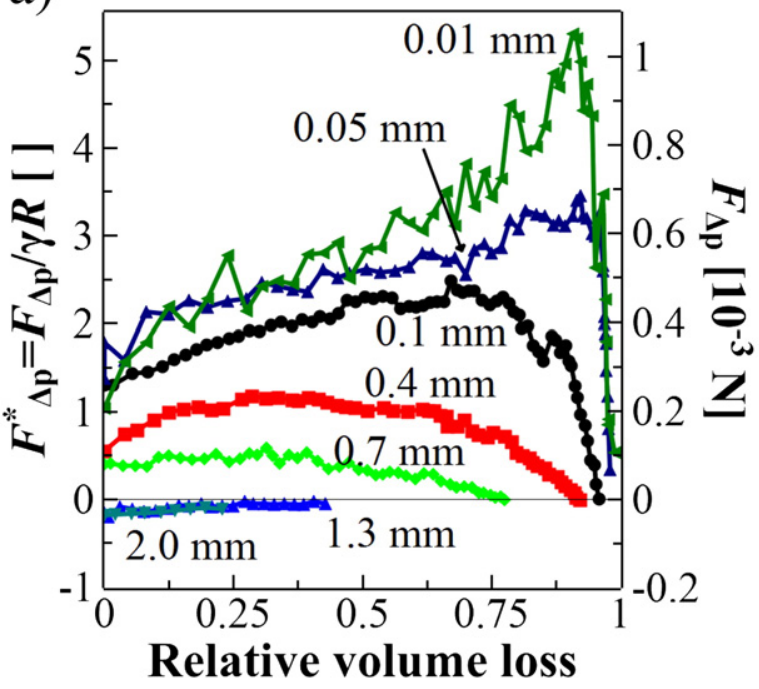

b)

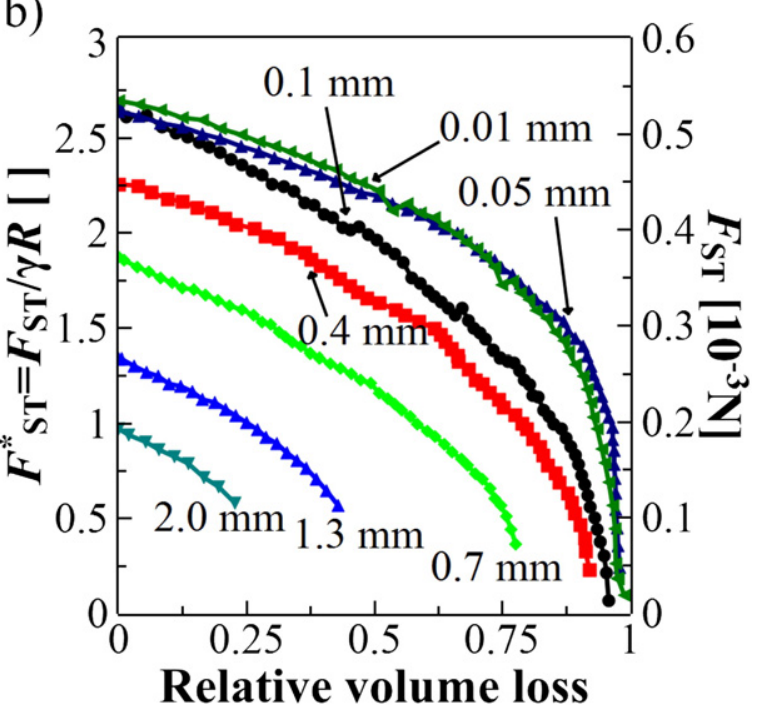

Fig. 7. Two components of capillary force during evaporation test: a) suction force, b) surface tension force.

For extension tests the initial negative $F_{\Delta \mathrm{p}}$ decrease with separation (except for $V=10 \mu \mathrm{l}$ ), and the terminal values are drastically reduced, to constant values slightly below zero (Fig. 8a), hence slightly repulsive eventually.

Surface tension force $F_{\mathrm{ST}}$ is proportional to the gorge radius and it decreases almost linearly with the increasing $D$, with only small acceleration near the end, and it is always attractive (Fig. 8b).

The initial and final values of both component forces for extension at different liquid volumes $V$ are shown in Fig. 9b.

\subsection{Total capillary force}

The evolution of the calculated total capillary force $F_{C A P}$ during evaporation is shown and compared to the directly measured force $F_{C A P-m}$ in Fig. 10a. $F_{C A P}$ and $F_{C A P-m}$ during evaporation are attractive all along. In their initial phase both exhibit a slight growth inherited from $\mathrm{F}_{\Delta \mathrm{p}}$ and hence $\Delta p$, with a modest maximum articulated for the smaller separations. The central phase is dominated by $\mathrm{F}_{\Delta \mathrm{p}}$, mainly for small separations, while in the terminal phase the trend of $\mathrm{F}_{\mathrm{ST}}$ prevails (for the considered volume of liquid).

a)

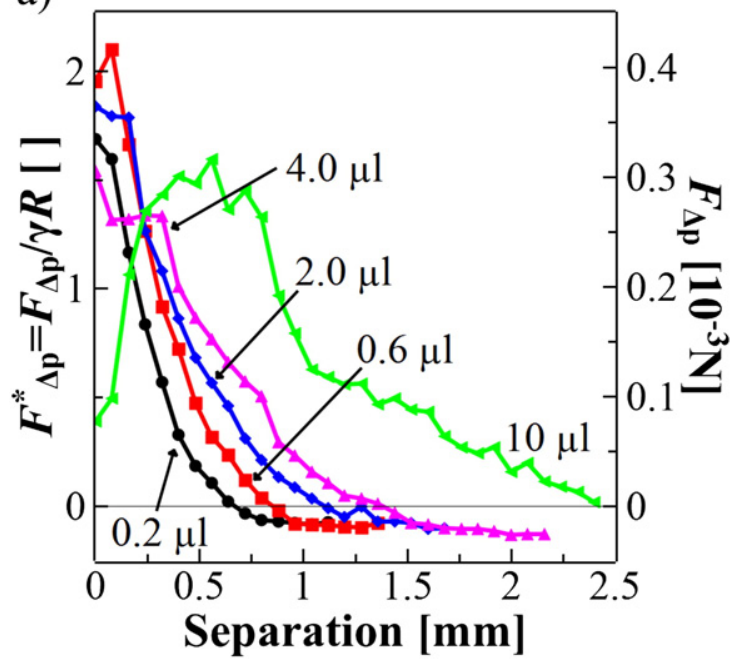

The comparison $F_{C A P}$ with the $F_{C A P-m}$ is more than good. This is an indirect confirmation that adopted experimental techniques are sufficiently accurate for the present purpose. When there is a divergence, $F_{C A P-m}$ is usually lower. A noticeable anomaly of the calculated forces is an inversion of the magnitudes of forces at extremely small separations of 0.05 and $0.01 \mathrm{~mm}$, and their relatively significant departure from the corresponding $F_{C A P-m}$. As obvious, these tests were repeated several times and the results appear to persist, suggesting that either our measurements at such small separations are biased, or there are shortdistance phenomena that interfere that we do not consider. However, based on the Molecular Dynamics simulations of the capillary bridges, both the separations and the contact areas tested here are still within the range of validity of Kelvin theory [23].

\section{Discussion}

\subsection{Laplace pressure evolution}

Prior to further considerations the following disclaimer is due. According to its definition Laplace pressure, $\Delta p$, is proportional to the mean curvature of the liquid/gas interface, which is deemed as constant,

b)

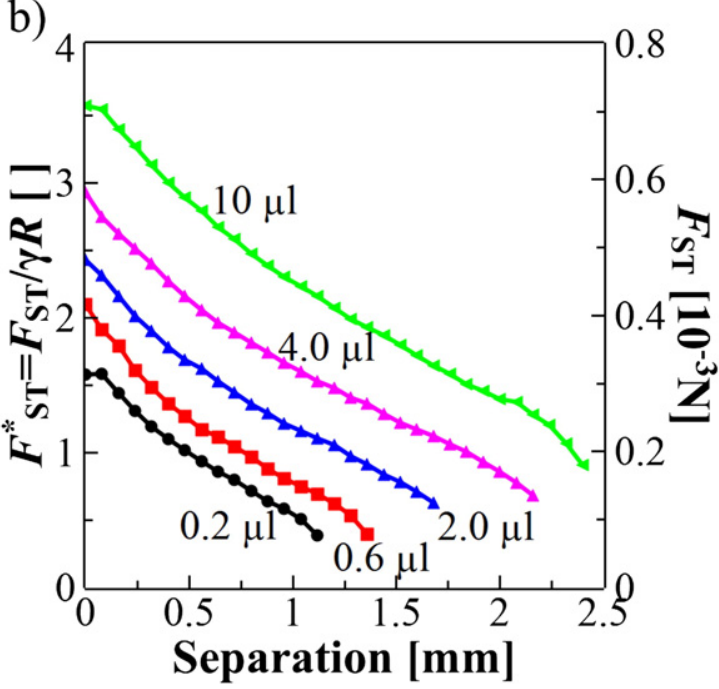



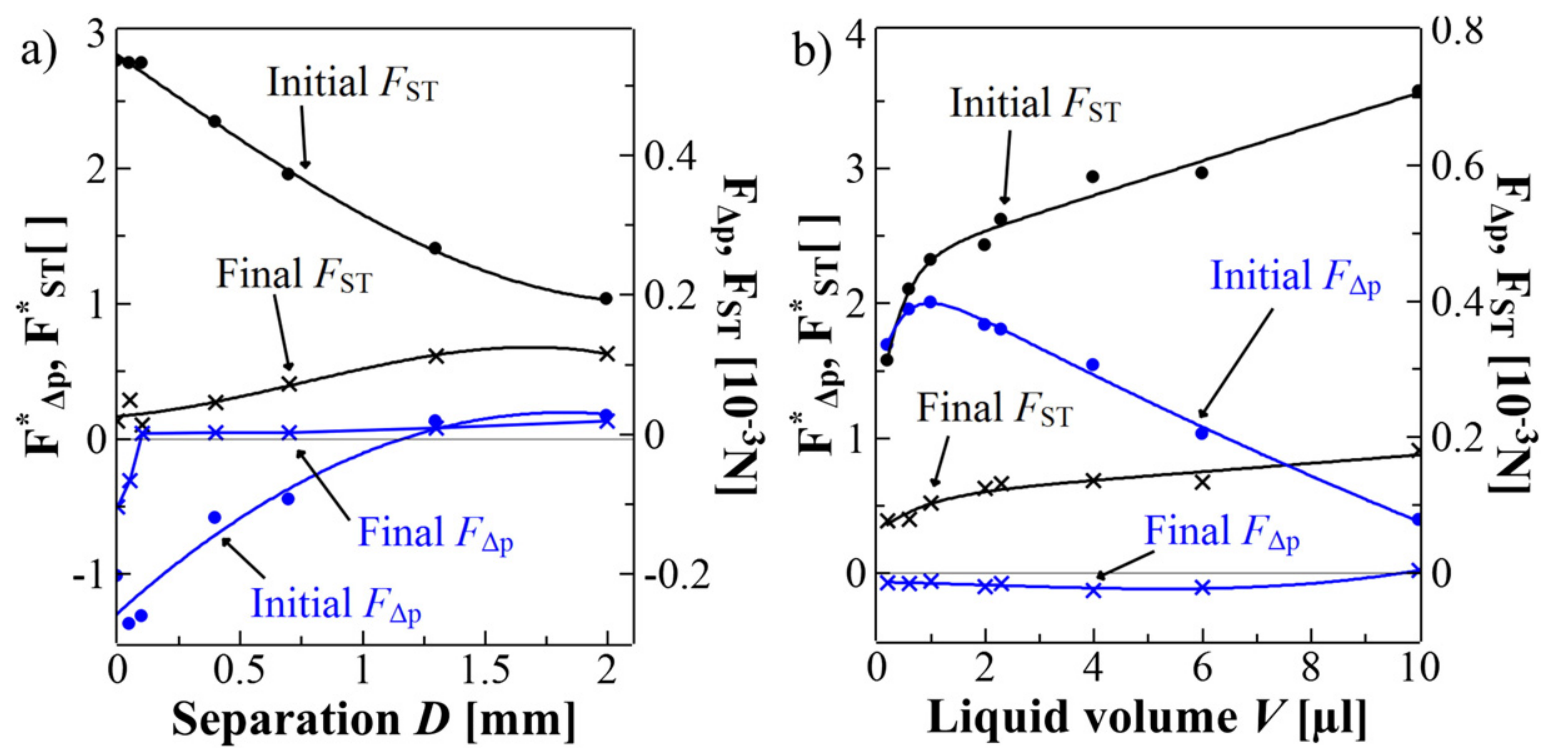

Fig. 9. Initial and final values of surface tension forces and suction forces for different separations at evaporation (a), and different liquid volumes at extensions tests (b).

hence enclosed within a Delaunay surface [24]. Hence, $\Delta p$ is understood as constant within the bridge body, thus implying no flow within the bridge. There are several observations suggesting several departures from these postulates. These include the observations of an actual flow of liquid within the bridge, intrinsic in the bridge extension process to accommodate the overall change of bridge shape, or in the case of evaporation to accommodate differentiated evaporation flux at various locations associated e.g. with the moving triple line [25-27]. Hence, $\Delta p$ in these considerations does not mean the actual fluid pressure (even assuming an atmospheric gas pressure), but a value calculated from the measured curvatures at selected cross-sections of the bridge body, under reasonable assumptions of usual symmetries.

Having said that, an indirect corroboration of $\Delta p$ calculations is provided by the comparison of $F_{C A P}$ calculated at the bridge gorge bridge crosssection perimeter with the corresponding $F_{C A P-m}$ measured from the same experiments [1]. The comparison shows very limited differences.

Setting the results of evaporation tests at constant $D$ and of the extension tests at constant $V$ within the same framework as shown in
Figs. 2, 3 and 6 indicates that neither $r_{g}, r_{e x t}, \Delta p$ nor $F_{C A P}$ constitute unique functions of $D$ and $V$, within the entire domain of these variables. That means that neither of these variables can be seen as a state function of $V$ and $D$ over their entire domain. The biggest departure from such a concept is seen in the range of small volumes and small separations. An expectation of a unique state function has been suggested in early studies [28]. However, more recent studies especially on contact phenomena in sessile drops [27] as well as the results presented here, suggest consistent departures from such an idealized concept.

In the previous work [1,2] we have found a relationship between the evolution of the measured capillary force $F_{C A P-m}$ and the evolution of two variables that are not part of Young-Laplace law, i.e. contact angle $(C A)$ and contact diameter $(C D)$. In particular, we concluded that there is a strong correlation between the capillary force and the events of pinning and de-pinning (as defined by de Gennes et al. [29]) of $C D$ and $C A$. In what follows we take a similar approach to Laplace pressure.

To start with, let us note that for evaporation a significant initial negative $\Delta p$ (suction) and also an initial attractive $F_{C A P}$ acting at the
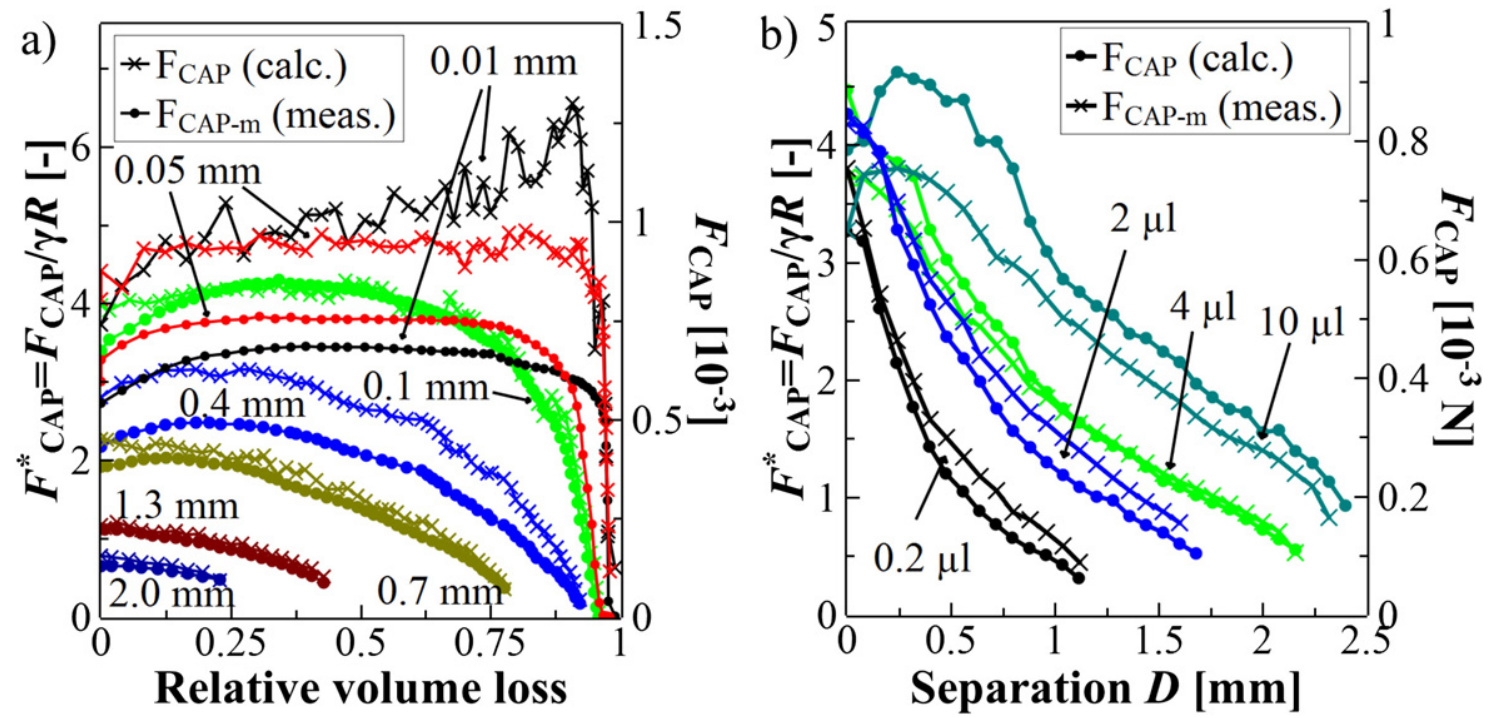

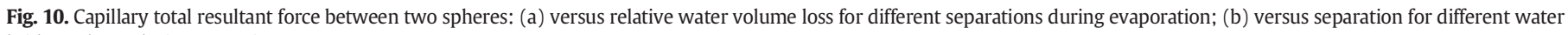
bridge volume during extension. 
grain contact, develop for the majority of the inter-particle distances tested, except for the most remote grains (separations 1.3 and $2 \mathrm{~mm}$ ). In the latter case $\Delta p$ is always positive (but small), and thus $F_{\Delta p}$ is repulsive. However, because of the presence of a larger surface tension force $F_{S T}$, the resultant force $F_{C A P}$ is still attractive, even in those cases.

In all the cases of different separations, the evolution of $\Delta p(V)$ exhibits one or more episodes of instability. We shall examine the evolution of the capillary pressure and the mentioned instabilities separately for what was previously identified as three geometrical categories of bridges of very short and very wide bridges (VSVW), short and wide bridges (SW) and tall and slim bridges (TS, see Table 1)

For short and wide bridges (SW) $\Delta p$ is initially negative (suction) and quite modest, about 10-12 Pa, but it can grow 4 to 8 times that during evaporation. The maximum suction is reached at $60 \%$ or $83 \%$ of the relative volume loss, for $D=0.7 \mathrm{~mm}$ and $D=0.4 \mathrm{~mm}$ respectively. After reaching the maximum, suction falls relatively fast, reaches zero and subsequently evolves into a positive pressure at which the bridge eventually ruptures.

For tall and slim bridges (TS) the initial $\Delta p$ is right away positive even if quite moderate, about 20-30 Pa, but it can grow, mainly at the last minute (literally) prior to rupture to 28 and $52 \mathrm{~Pa}$ for separations of $1.3 \mathrm{~mm}$ and $2 \mathrm{~mm}$, respectively.

For very short and very wide bridges (VSVW) $\Delta p$ is initially negative (suction) and similar to that for SW bridges (about 10-12 Pa). However the suction growth, especially after half of the water evaporated becomes incomparably more dramatic, reaching the value up to $10 \mathrm{kPa}$ at $D=0.01 \mathrm{~mm}$. This value is two orders of magnitude higher than the highest value for SW bridges. Whether or not this value falls in an unstable fashion back to zero, or to positive values, remains unknown, as no sufficient resolution images could be obtained, not even with a fast-camera, at such small distances. For all the cases, when images were available, the bridge rupture occurs at a positive pressure.

During the bridge extension tests Laplace pressure evolution $\Delta p(D)$ presents similar main characteristics, that is, from an initial suction it converts into terminal positive pressure. However, details of the evolution are significantly different from those during evaporation. The average terminal positive $\Delta p$ at rupture through extension is about twice as high as that at the rupture due to evaporation. The values of $D$ at the moment of conversion from suction to positive $\Delta p$ changes range widely from $D=0.6 \mathrm{~mm}$ to $D=2.1 \mathrm{~mm}$. So, we cannot say that there is a characteristic extension of the bridge at rupture. However, the smaller is the volume of the bridge, the smaller is the distance at which it ruptures, and consistently higher is the value of the pressure at rupture. Interestingly, during evaporation, the larger the separation (or the distance), the higher the positive pressure at rupture. Even more intriguing is the fact that the last-second pressure pulse (calculated from the fast camera images) is significant, but there, the larger the separation the smaller the pulse. Notably, at the smallest separation for which the fast camera provided usable images ( $D=0.4 \mathrm{~mm}), \Delta p$ reached $2 \mathrm{kPa}$ at the end of evaporation. An analogous pressure impulse at the end of extension had also an inverse tendency to the prior continuous evolution, and the bigger the liquid volume, the bigger the pressure pulse.

The presence of a positive pressure in the terminal phase of both evaporation and/or extension implies that in that phase there is repulsion exerted by water against the grains, rather than attraction. On the other hand, the value of that repulsion force is small compared to the

\section{Table 1}

Geometrical types of bridges, initial configuration.

\begin{tabular}{lll}
\hline Type of bridge & Acronym & Separation $D$, at $V_{\text {initial }}=4 \mu \mathrm{l}$ \\
\hline Very short and very wide & VSVW & $D<0.1 \mathrm{~mm}$ \\
Short and wide & SW & $D=0.1,0.4,0.7 \mathrm{~mm}$ \\
Tall and slim & TS & $D>0.7 \mathrm{~mm}$
\end{tabular}

initial value, mainly because in both cases the surface area, across which the pressure acts, becomes nearly zero.

Nevertheless, the resultant total capillary force, initially being attractive, remains such until rupture, because of the surface tension force component $\mathrm{F}_{\mathrm{ST}}$ which is always attractive and larger than the pressure component $F_{\Delta p}$, as seen in Fig. 9a and b.

In no case, a significant increase of attractive force was measured near bridge complete dewatering, which would suggest an increased apparent cohesion.

\subsection{Instabilities}

As mentioned before, Laplace pressure evolution during evaporation of two-grain capillary bridges exhibits several features that may be perceived as instabilities. Similar instabilities were seen in multi-grain evaporating bridges [30]. They appear to play a fundamental role in fracture of drying granular media [31].

For SW bridges there are four such consecutive instances.

The first instability, in SW bridges coincides with the point of maximum suction reached quite early in the process, which initiates either a relatively gentle (for $D=0.7 \mathrm{~mm}$ ) or faster (for $D=0.4 \mathrm{~mm}$ ) demise of suction and generation of positive pressure. Following that, the liquid bridge undergoes two Rayleigh-type instabilities, to be further subject to a pinching instability leading eventually to rupture, way before water evaporates completely. An obvious question is about what causes each of the instabilities, and what would be criteria that would allow predicting the occurrence of each of the events.

Laplace pressure $\Delta p$ by its definition is proportional to the mean of two curvatures of the bridge external surface, $\kappa_{\mathrm{g}}$ and $\kappa_{\text {ext }}$. It is also the evolution of the mean of these two curvatures, what determines the fate, and hence presumed instabilities of $\Delta p$. In particular, if there is suction developing within the capillary bridge, it is due to the negative $\kappa_{\text {ext. }}$. Fig. 11 shows the evolution of $\kappa_{\mathrm{g}}$ and $\kappa_{\text {ext }}$ as a function of the relative volume loss (for evaporation) or of the separation (for extension).

It is noticed first, that during evaporation the two curvatures are consistently of the same order of magnitude for most separations, but of the opposite sign. Only for the smallest separations, the negative $\kappa_{\text {ext }}$ becomes an order of magnitude higher than $\kappa_{\mathrm{g}}$, and only at the final moments, hence leading to a spike of suction at that distances followed by a terminal conversion of $\Delta p$ into positive values. The conversion could not be captured at such small separations, because of the resolution of the images.

It appears that during the initial stage of evaporation, but also through the mid-stage of the process when the gorge radius is still large, it is the negative $\kappa_{\text {ext }}$ that dominates and increases, thus producing an increasing $\Delta p$ (suction). Clearly, by definition of $\Delta p$, its instability onset, defined as $d \Delta p / d V=0$, implies an equality of the rates of the change of both curvatures. Indeed, for the mid-separations $(D=$ $0.4 \mathrm{~mm}$ and $D=0.7 \mathrm{~mm}$ ) the observed, relatively marked maximum and instability of suction appears to be associated with a sudden surge in $\kappa_{\mathrm{g}}$ (or sudden $r_{\mathrm{g}}$ decrease) while the increase in $\kappa_{\text {ext }}$ growth is more gentle. However, the underlying question remains: what controls the change in the rate of the bridge curvature in the process of liquid evaporation.

In our recent study of evolution of the capillary force due to evaporation [1] it was found that onset of the decrease in the capillary force was closely correlated with two crucial characteristics of the bridge evolution: depinning of the gas-liquid-solid contact ("triple") line, hence the onset of the decrease in the contact diameter $C D$, and subsequent its re-pinning, or the arrest of the decrease of the contact angle $C A$ [28]. Pinning and depinning of contact line is a central issue in studies of evaporation of sessile drops $[27,32,33])$ and the evolution of their shape. To analyze possible analogous correlations, $\Delta p$ and the two contributing curvatures $\kappa_{\mathrm{g}}$ and $\kappa_{\text {ext }}$ against the corresponding current volume of water $V, C A$ and $C D$ are presented in Figs. 12a) and 13a) for the two mid-range separations $D=0.4 \mathrm{~mm}$ and $D=0.7 \mathrm{~mm}$, 
a)

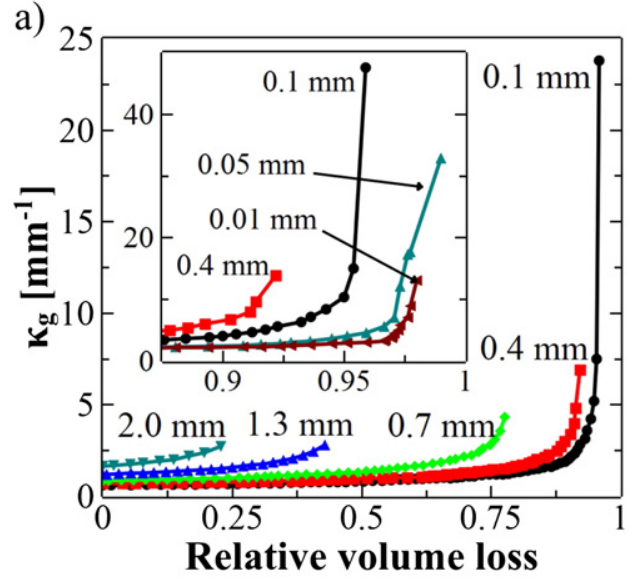

b)

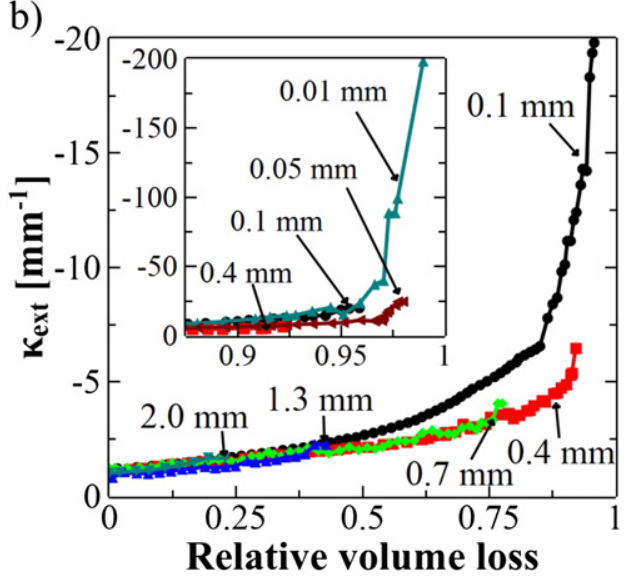

d)

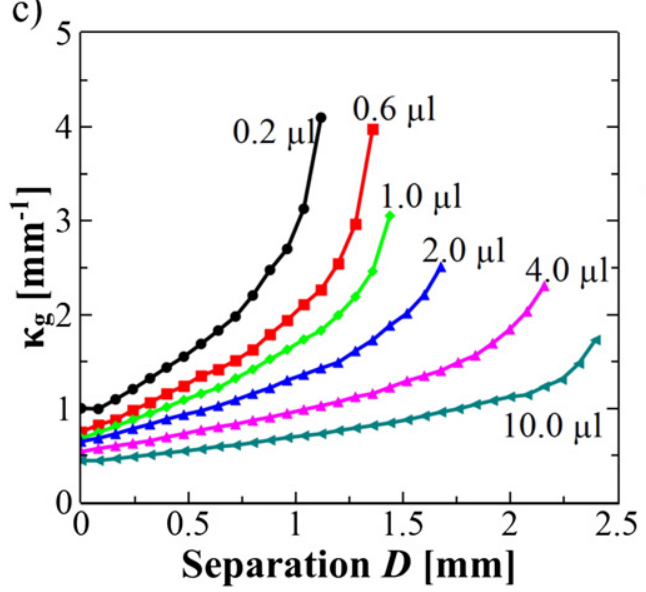

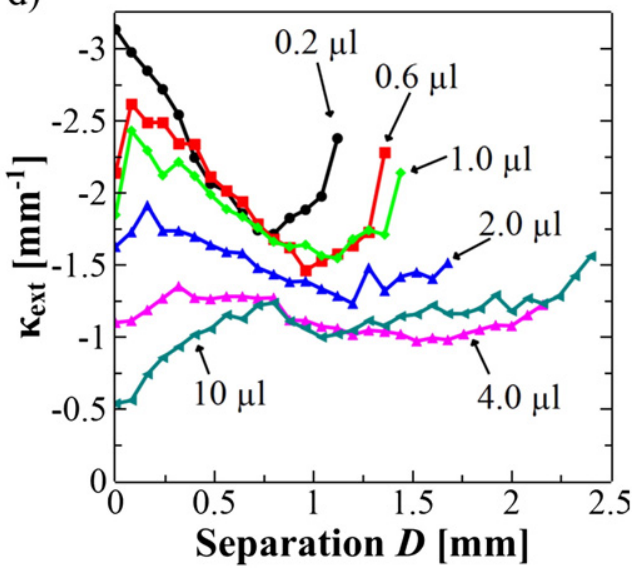

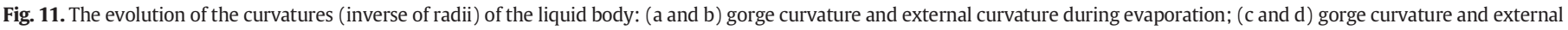
curvature during extension.

together with their rates versus liquid volume $\delta \Delta p / \delta V, \delta \kappa_{g} / \delta V, \delta \kappa_{\text {ext }} / \delta V$ (Figs. 12b, 13b).

It must be clarified at this point that from sessile drop studies it results that the actual (measurable) $C A$, is not a constant and is not the equilibrium $C A$, as in Young-Laplace theory [34], but is a variable quantity, in our cases changing between $28^{\circ}$ and $9^{\circ}$. In what is presented in the following (see also [1]), it is measured as so called "apparent $C A$ " determined at some distance from the "triple" line [33].

Interestingly, $\kappa_{\mathrm{g}}$ (and $r_{\mathrm{g}}$ ) changes relatively smoothly, in contrast to $\kappa_{\text {ext }}$ (and $r_{\text {ext }}$ ), which exhibits a large number of stick-slip events. The later seem to be inherited from the observed evolution of both $C A$ and $C D$. Such behavior of the triple contact has been observed since long, especially in the evolution $C A$ but also in $C D$ of evaporating sessile drops $[35,36]$.

For $D=0.4 \mathrm{~mm}$, five phases of the process can be identified in terms of correlation of $\Delta p, \kappa_{\mathrm{g}}$ and $\kappa_{\text {ext }}$ to the evolution of $C A$ and $C D$.

Phase 1 : in the volume range of 4 to $2.3 \mu \mathrm{l}, C D$ is pinned while and $C A$ decreases from $33^{\circ}$ to $16^{\circ}$, coincidently also $r_{g}$ does not change in that phase. This implies only a very modest increase in negative $\Delta p$ to reach $24 \mathrm{~Pa}$, resulting from a (modest) change in external radius, $r_{\text {ext }}$.

Phase 2: $C D$ becomes de-pinned, but $C A$ becomes pinned, while both curvatures accelerate with a concomitant increase in suction. As $\kappa_{\text {ext }}$ climbs at the same rate, $\kappa_{\mathrm{g}}$ starts accelerating right after the first $C D$ depinning, and takes over around $C D=1.5 \mathrm{~mm}$ (Fig. 12b). The point of the matching rates of curvatures marks the suction maximum and onset of the first (pressure) instability, i.e. onset of decreasing suction.
This coincides with the triple line arrest (both $C A$ and $C D$ pinned). Consequently, the continuing decrease of water volume imposes on the water body a new pattern via a change in $r_{g}$ rate (much faster) and $r_{\text {ext }}$ rate (slower), see Fig. 12b, and hence a drop in negative $\Delta p$, leading to Phase 3 . In contrast to the $r_{\text {ext }}$ (and $\kappa_{\text {ext }}$ ), which exhibits a large number of stick-slip events in correspondence to stick-slip behavior of $C A, r_{g}$ (and $\kappa_{\mathrm{g}}$ ) changes relatively smoothly. In other words, $\kappa_{\text {ext }}$ is much more sensitive to the changes of $C A$ at the triple line, than is $r_{g}$, which is directly driven by the decrease of liquid mass and changes in $C D$.

Phase 3: In this phase, characterized by negative $\Delta p$ and $\delta \Delta p / \delta V<0$, one see $r_{g}$ to decrease very fast. Once the absolute values of the two radii (and curvatures) become equal, best seen in Fig. 12a (middle image), according to Plateau's nomenclature, the free liquid surface evolves from nodoid $(\Delta p<0)$ into catenoid $(\Delta p=0)$, with $\Delta p$ developing further into positive values (and the surface transitioning from catenoid into unduloid, see Fig. 14a and [37]. This ( $\Delta p$ reaching zero) constitutes a second pressure instability. Physically that implies that the grains after Phase 3 are under a repelling pressure each other, rather than attracting. A posteriori review of the individual shots near $\Delta p=0$ (taken every 1 minute, regular camera) revealed no actual instability, while the recorded shape does not coincide with the mathematical form of catenoid surface, as seen in Fig. 14a (at the measured $\mathrm{kg}_{\mathrm{g}}$. However, it may be that a fast-camera recording $(20,000$ shots/sec) would be necessary to capture this particular transition, as it is the case in Phases 4 and 5 (see below). 
a)

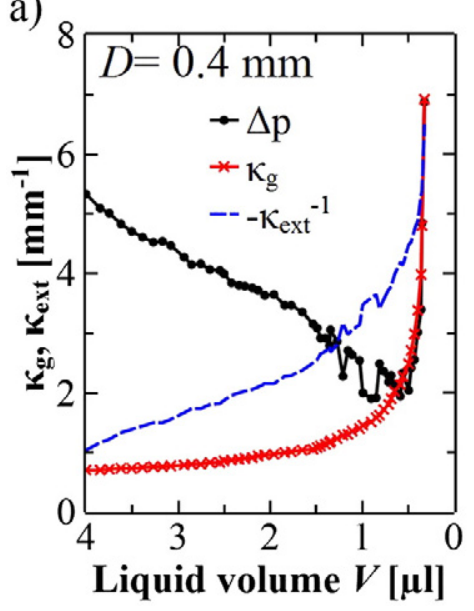

b)

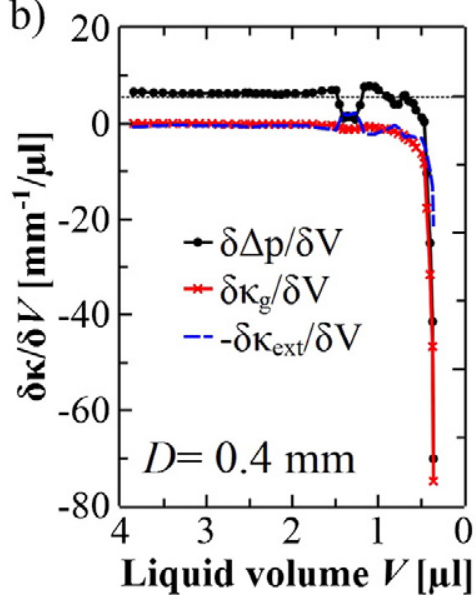

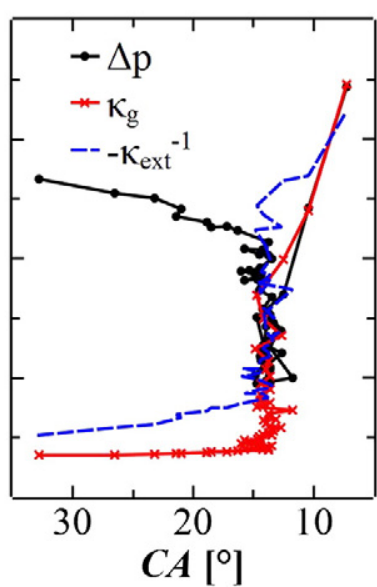
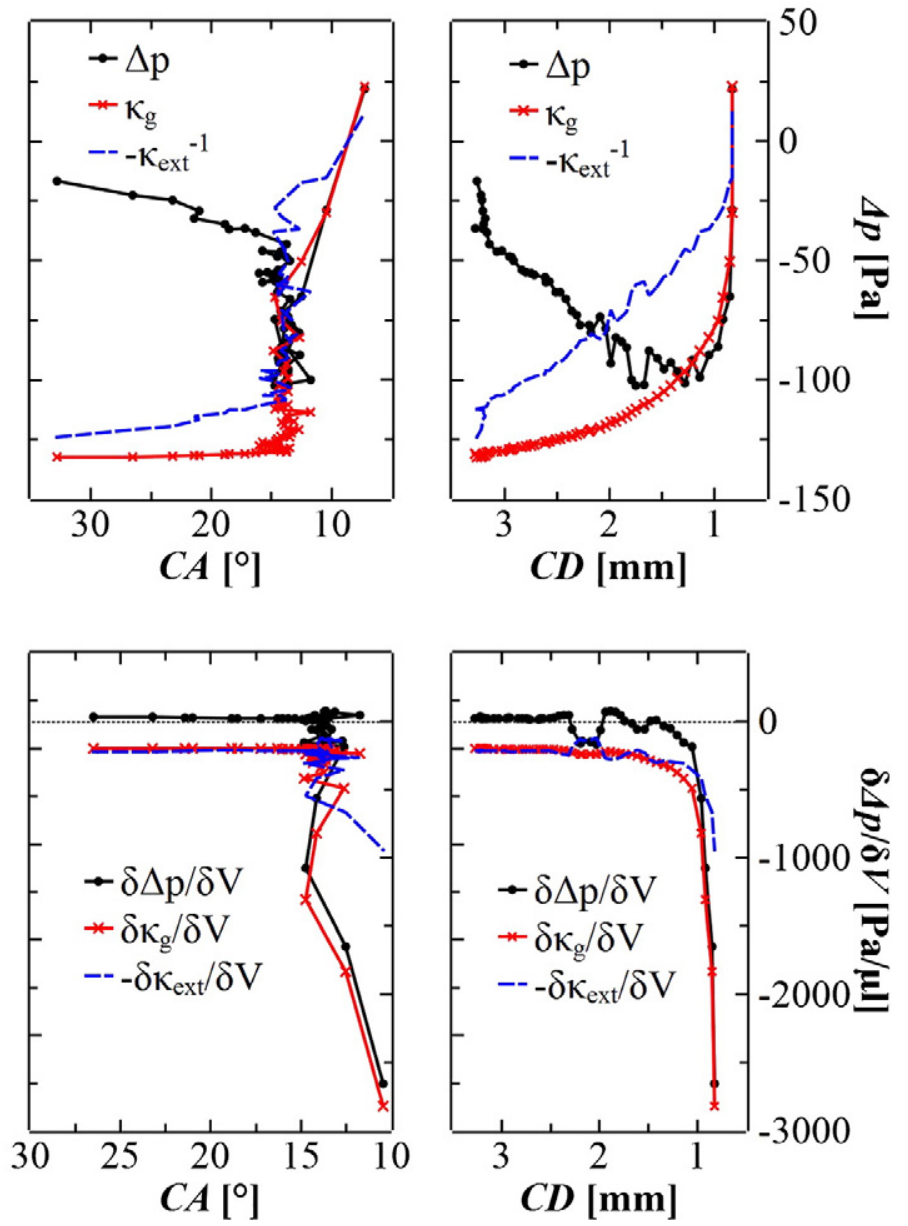

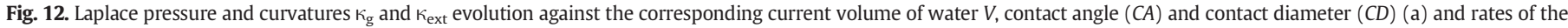
curvatures and Laplace pressure (b) for the separation $D=0.4 \mathrm{~mm}$.

Phase 4: In this phase a positive pressure builds up within the bridge, while the bridge still maintains the form of unduloid (Fig. 14a,b, left), with a rate faster than the previous process, but yet within seconds, a third instability takes place at positive pressure and consists in the transition from an unduloid into a cylindrical waterwire. The transition (Fig. 14b-d, see [2]) occurs about $0.5 \mathrm{sec}$. prior to the eventual rupture, at $r_{\mathrm{g}}$ becoming smaller than about $0.1 \mathrm{~mm}$ as captured with the use of fast-camera, allowing to calculate Laplace pressure for the first time. The pressure appears to be in a well contained range between 50 - 300 Pa for all tested separations (Fig. 15). The formation of the water-wire occurs within $0.0003 \mathrm{sec}$. Interestingly, over the period of the wire formation, its radius is almost constant, while its height increases displacing away the masses of liquid below and above it, taking the shape of two cones. (Fig. 14a,b,c and e).

Transition from unduloid into water-wire is well known since the original work of Plateau [38], on liquid stream instabilities, see Fig. 14d, and has been discussed by Padday et al. [39] and Peregrine et al. [40], for bridges being extended, or pendant drops, for but never before in the context of evaporation of a bridge between two spheres. Weeks et al. [41] reported Laplace pressure calculated from images of AFM tip-plate bridges through Environmental Scanning Electron Microscope for various relative humidity levels. Phase 5 consists in the evolution of the cylindrical liquid-wire, with is length growing, while the volumes below it and above it convert into conical bodies in contact with the grains. The process terminates with a fourth instability consisting of pinching that takes place most commonly simultaneously at the wire extremities (see Fig. 14e and [2] for details). Pinch-off is a well- known phenomenon in selfsimilar fluid instabilities (see e.g. [42]). It is worth noting that $\kappa_{\mathrm{g}}$ is positive, nearly constant and very high along the height of the entire cylinder, whereas $\kappa_{\text {ext }}$ (initially negative) becomes zero. Above the wire, the external curvature of the nearly conical surface $\kappa_{\text {con }}$ is also zero (see Fig. 14e). At the point of contact between the cylinder and the cone, there is a local singularity of a high curvature external curvature $\left(\kappa_{C}\right)$ in transition from zero of the column to zero of the cone. The resulting heterogeneity of the local fluid pressure may suggest liquid flow, in addition to the liquid instabilities.

It needs to be stressed that similar mechanisms and indeed phases to those presented above are observed for the liquid bridges in a similar range of separation of, i.e. $0.7 \mathrm{~mm}$. It appears that indeed in both $(0.4$ and $0.7 \mathrm{~mm}$ ) cases $\kappa_{\text {ext }}$ are consistently and increasingly higher than $\kappa_{\mathrm{g}}$, consequently resulting in the initially increasing suction. This is enhanced by the fact that initially $\kappa_{\mathrm{g}}$ is changing very little.

Simultaneously, over that range of $C A$ drop from $33^{\circ}$ to $13-15^{\circ}, C D$ is pinned (constant). At the same time, over the initial volume loss from 4 to $3 \mu$, there is very little $(<1 / 10$ th for $D=0.4 \mathrm{~mm}$ and $<1 / 5$ th for $D=$ $0.7 \mathrm{~mm}$ ) of $r_{g}$ change, and over half of the volume loss, there is a still small change in $\kappa_{\mathrm{g}}$, and as long as $C D$ is pinned, the $C A$ decrease, is the main geometrical change of relevance of the water body. However, 

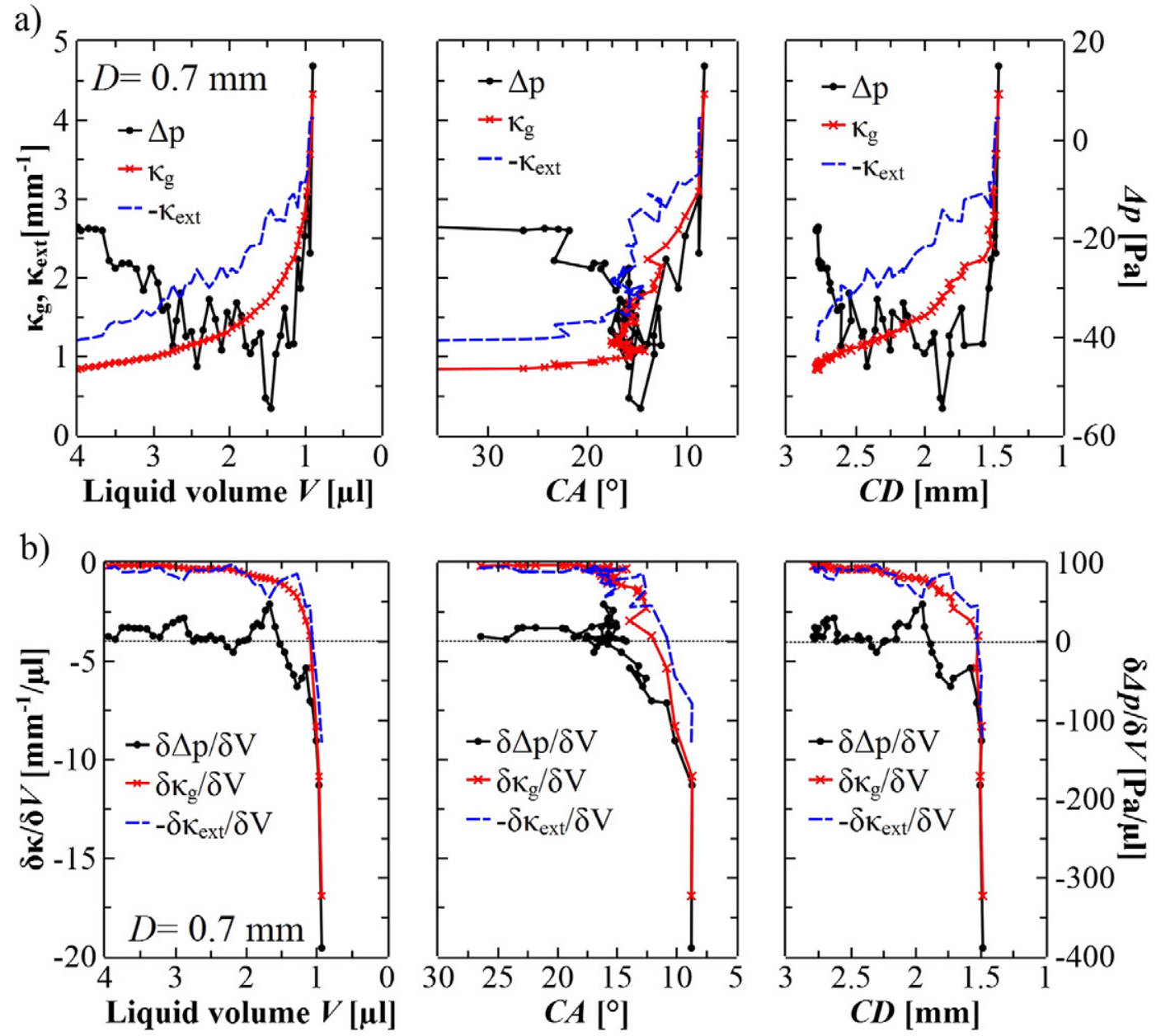

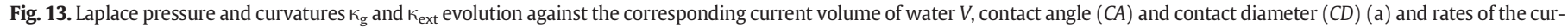
vatures and Laplace pressure (b) for the separation $D=0.7 \mathrm{~mm}$.

a)
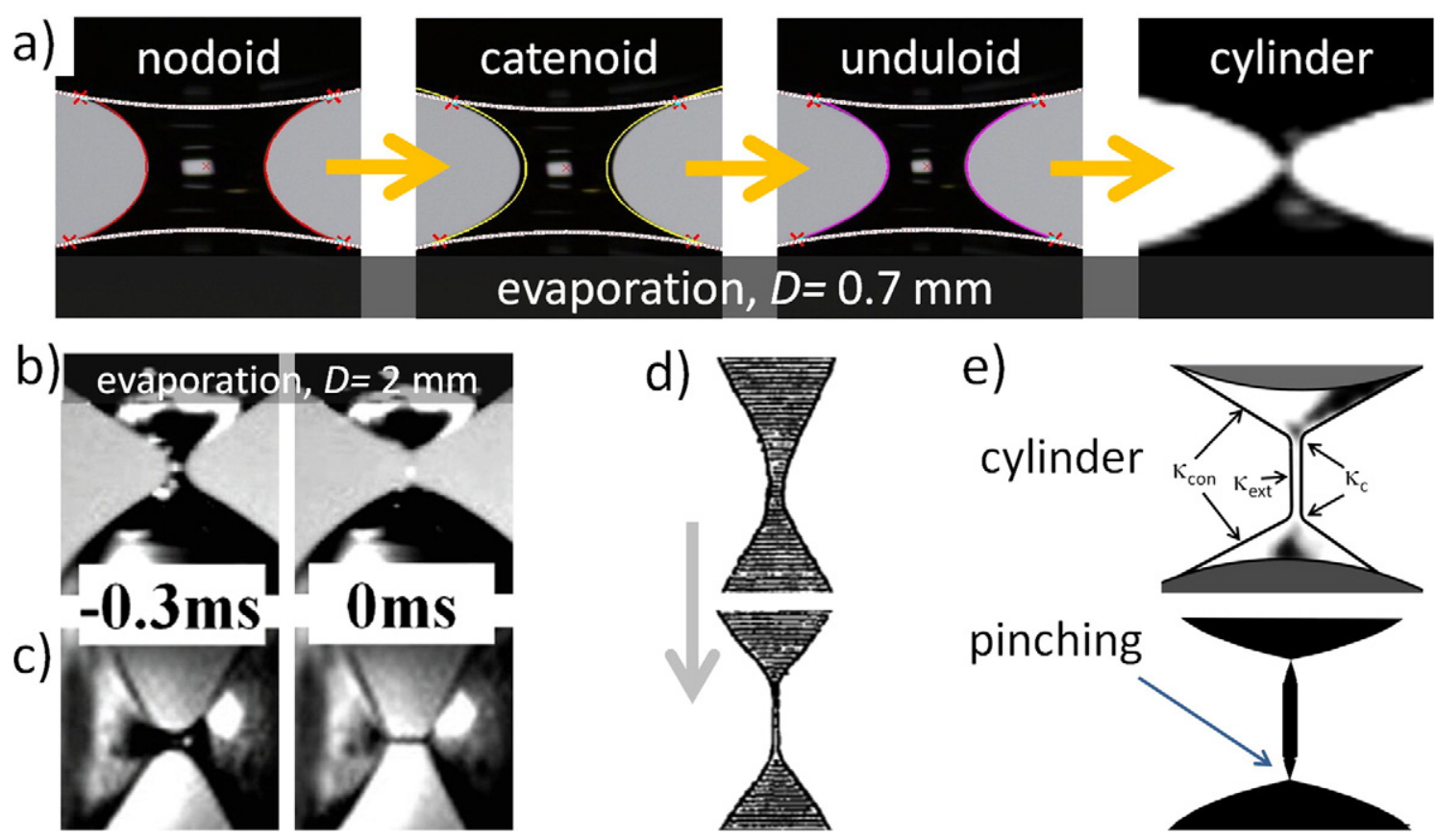

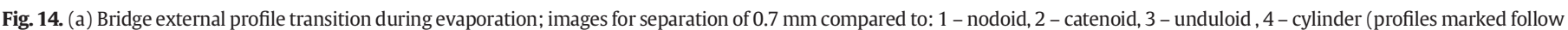

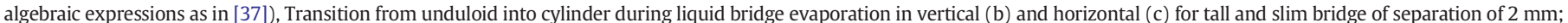

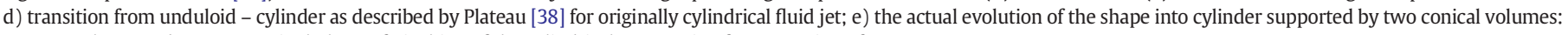
top-an early stage; bottom-terminal phase of pinching of the cylindrical water-wire, for separation of $2 \mathrm{~mm}$. 

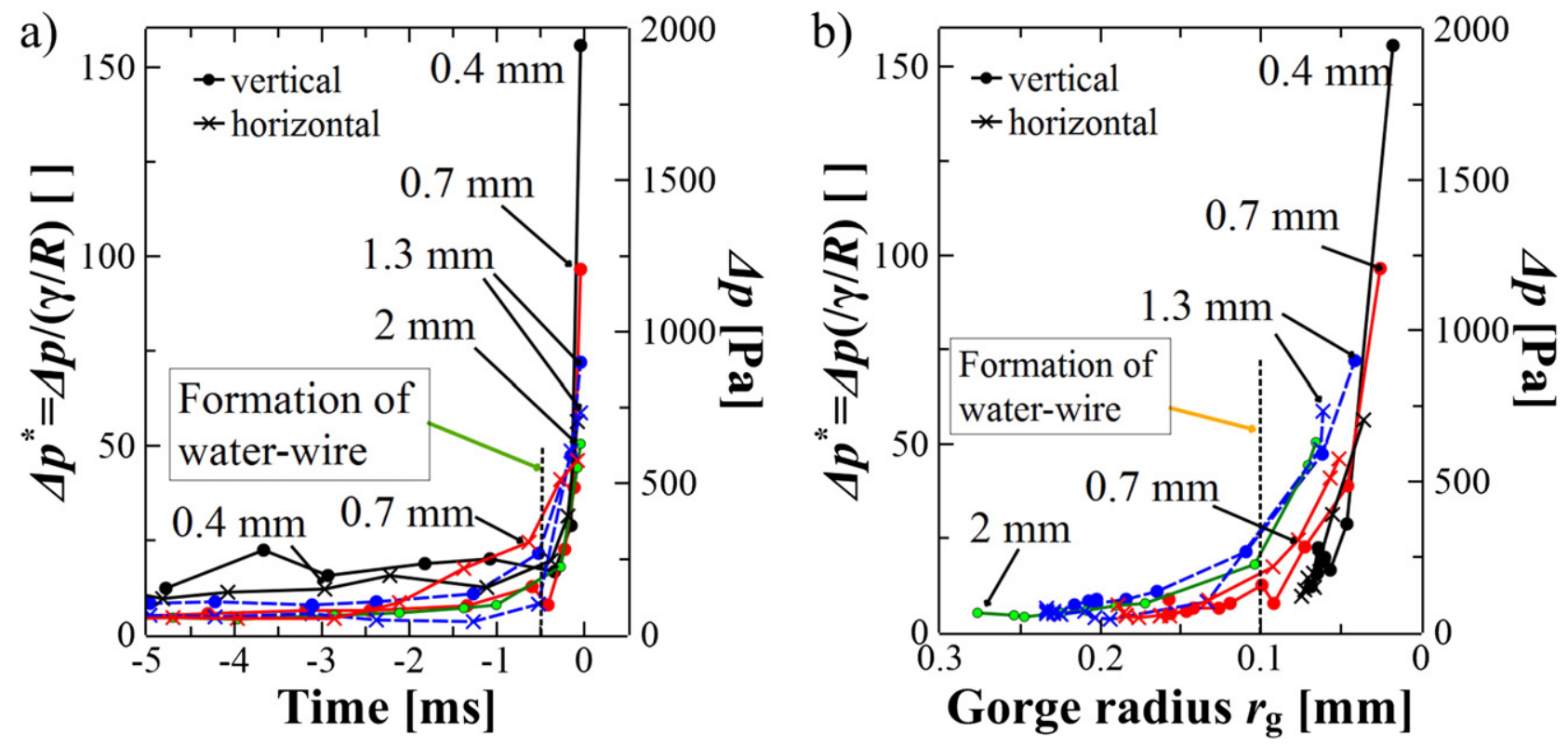

Fig. 15. Evolution of $\Delta p$ before the rupture: against time (a) and against gorge radius (b).

over the same range, $\kappa_{\text {ext }}$ does increase. All these changes are very clear in the lateral profile evolution shown for $0.7 \mathrm{~mm}$ in Fig. 16a.

Consequently, the difference between the curvatures increases, and so does suction. The rates of $\delta \Delta p / \delta V$ are practically constant till $62.5 \%$ (for $D=0.4 \mathrm{~mm}$ ) and $35 \%$ (for $D=0.7 \mathrm{~mm}$ ) of water loss. After that threshold, both rates abruptly increase, with $\kappa_{\mathrm{g}}$ growing eventually faster (see Figs. 12b, 13b). The onset of the rate growth of the gorge curvature starts the slowing of the suction growth, which therefore can be viewed as a precursor for instability, that occurs when the two curvature rates become equal, marking the suction maximum. Note that Figs. $12 \mathrm{~b}$ and $13 \mathrm{~b}$ clearly show that for $D=0.4 \mathrm{~mm}$ and for $\mathrm{D}=$ $0.7 \mathrm{~mm}$ the gorge curvature (and radius) accelerate, while the contact diameter is slowing down to eventually become pinned. Further, $\kappa_{g}$ value becomes larger than $\kappa_{\text {ext, }}$ and at that point suction decreases to zero, and positive pressure starts to build up. The zero pressure point is slightly preceded by re-pinning of the contact line.

It can be speculated at this point that for the reasons to yet be clarified during evaporation $\delta C D / \delta V$ slows down significantly around $C D=2.2$ to $1.5 \mathrm{~mm}$ (68-46\% of the original diameter) in the considered cases, to be eventually re-pinned down at, respectively, $C D=1.5$ and $0.8 \mathrm{~mm}$ ( $46 \%$ to $24 \%$ ). The continuously progressing water volume loss at roughly constant rate, in response to the pinning of $C D$, induces an acceleration of $r_{g}$ decrease and hence of $\kappa_{\mathrm{g}}$ increase. At some point, $\kappa_{\mathrm{g}}$ becomes the larger of the two curvatures, hence leading to the conversion from suction to pressurization of the bridge. In such a case, it all appears to be the play of geometry, with the contact line pinned, and $r_{g}$ intensely decreasing, as seen in Fig. 5, Mielniczuk et al. [1], while the pressure evolution is a resulting development.

For TS bridges, as indicated earlier, $\Delta p$ is always positive, and for large part of the process does not change much, as also $r_{e x t}$ does not change. The positive $\Delta p$ implies that $\kappa_{\mathrm{g}}$ is higher than $\kappa_{\text {ext }}$, see Fig. 17a,b and [1]. The contact diameter does not change much either (pinned), so the two geometrical characteristics that do change to accommodate the volume decrease are $C A$ (especially for $1.3 \mathrm{~mm}$ ) and $r_{g}$. As with SW bridges, at some point the contact line gets re-pinned and as a result $\kappa_{\mathrm{g}}$ starts to grow much more aggressively. This marks the point of pressure instability. Clearly, there is no Rayleigh-Plateau instability (zero pressure transition), as pressure is positive from the very beginning. However, the instability connected to the formation of the water-wire and that to the terminal pinching are well articulated, as visualized in Fig. 16b.

VSVW bridges exhibit during evaporation several features differentiating them from SW bridges. First, curvatures, especially $\kappa_{\text {ext }}$, may be up to ten times higher than in SW bridges. Consequently, suction also is much higher, reaching nearly $10 \mathrm{kPa}$ for $D=0.01 \mathrm{~mm}$. So, this is the only case in the entire spectrum of separations in which $\Delta p$ reaches significant negative values (suction). However, that happens at the advanced stage of the process when the area over which such suction
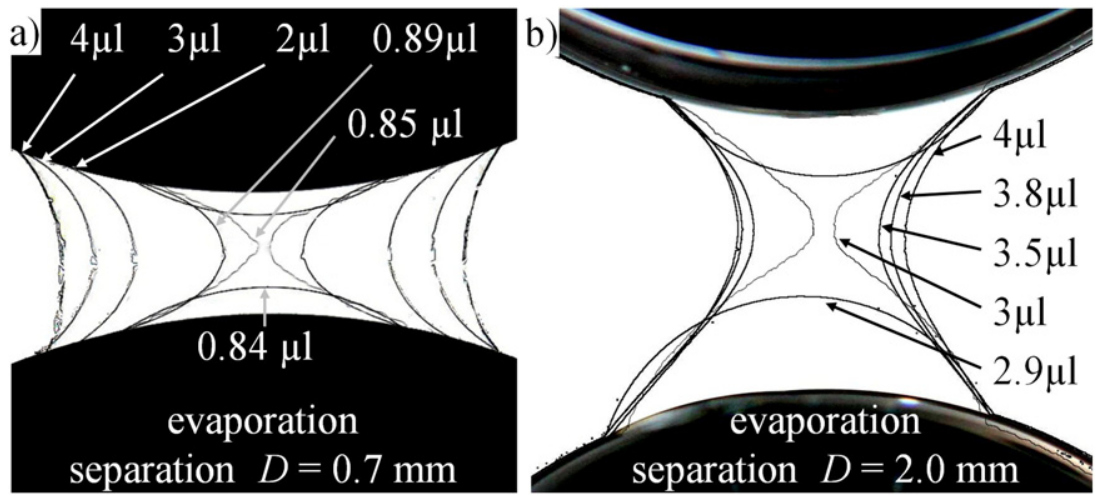

Fig. 16. Evaporation of liquid bridge from initial volume $V=4 \mu$, and separation $D=0.7 \mathrm{~mm}$ (a) and $D=2 \mathrm{~mm}$ (b). 

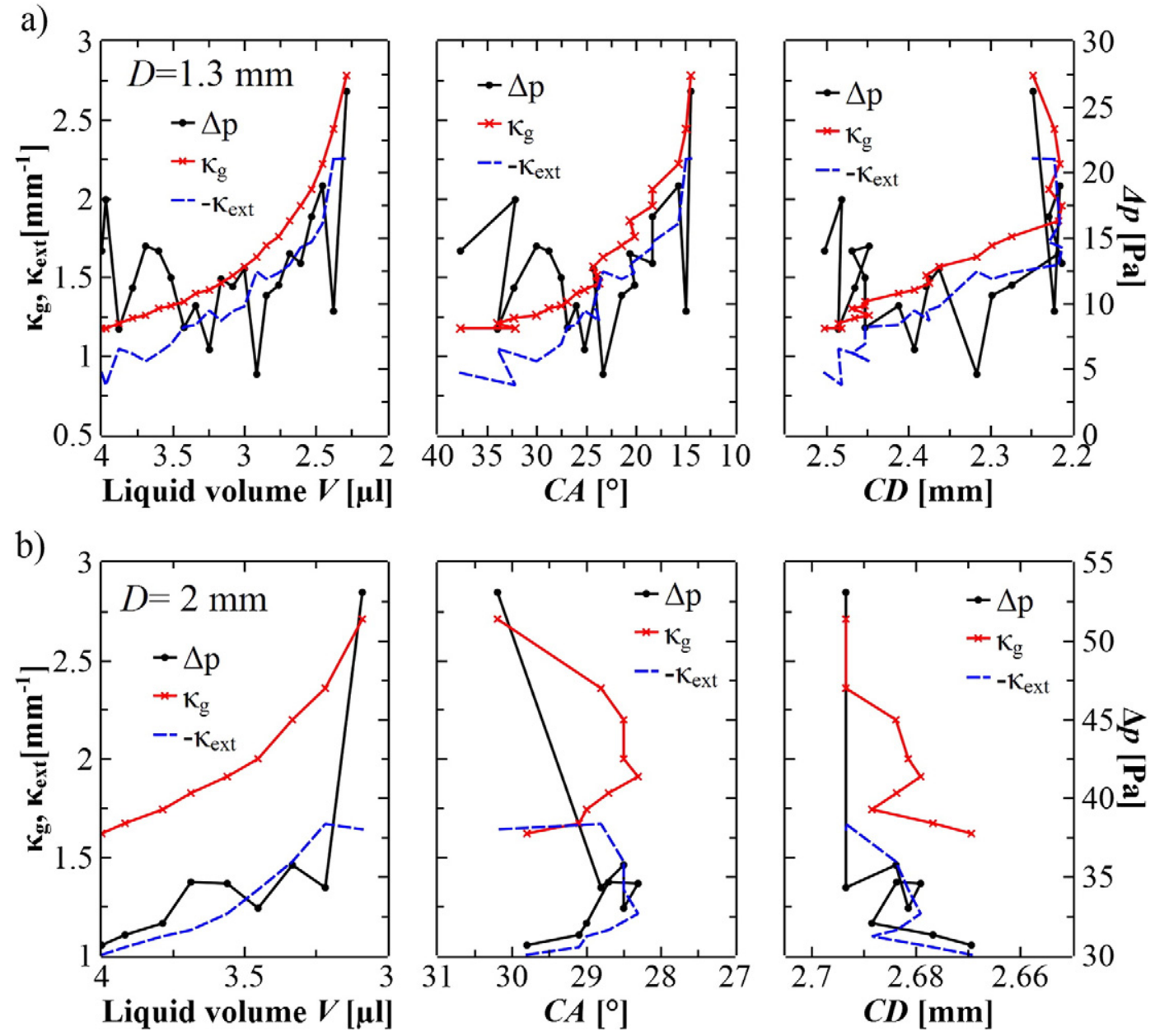

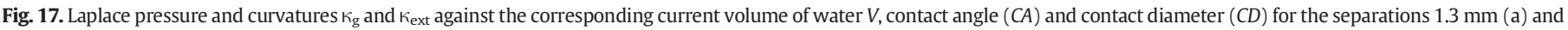
$2.0 \mathrm{~mm}$ (b).

acts is of the order of $10^{-5} \mathrm{~mm}^{2}$ rendering the adhesion force miniscule. In two of the cases, of slightly larger separations $(0.1$, and $0.05 \mathrm{~mm})$ it was possible to capture the final suction peak, and its post-peak (instable) fall either to nearly zero values, or even beyond into positive values. Interestingly, in all three cases investigated, $C A$ exhibits quite erratic behavior of stick and slip, but with a general tendency of increasing in value, from $15-40^{\circ}$, as opposed to larger separation bridges, for which $C A$ consistently decreases during evaporation. The onset of instability, as for the other separations, coincides (when observed) with a repinning of $C D$ and freezing of $C A$.

An essential observation in the context of the instabilities is that for VSVW bridges, no water-wire transition could be seen with the use of our measuring technique. Instead, a well articulated unduloid shape persists until its central part evaporates and the bridge breaks into two volumes of water. Thus, the two other instabilities, water-wire formation and its final pinching do not take place for this bridge category.

\subsection{Extension}

It should be emphasized that in the companion extension tests for bridges at a constant liquid volume, several fundamental similarities, but also differences in $\Delta p$ evolution and instabilities have been observed and compared to evaporation tests. The main difference is noticed in the evolution of $\kappa_{e x t}$, which is distinctly non-monotonic, of a higher average value, and of a more limited variability (see Fig. 12d). As a consequence of that and the monotonic increase of $\kappa_{\mathrm{g}}$, the resulting initial suction is much more dispersed for various liquid volumes, but its dominant trend is a decrease, with a much earlier transition into positive pressure values. The terminal $\Delta p$ at rupture are consistently higher than for evaporation. The initial pressure instability is present much earlier in the process than during evaporation. The other types of instabilities, as elaborated more in detail by Mielniczuk et al. [2] (i.e. formation of the water-wire), do occur for larger volumes of water $(>4 \mu \mathrm{l})$ with the following terminal wire pinching (symmetric, or non-symmetric), but are missing for smaller volume bridges. The phenomenon of the $C D$ re-pinning appears to be similarly linked to the first (pressure) instabilities, as in the evaporation tests.

\subsection{Context of earlier studies}

The intergranular force instability, water-wire formation and its instability have been observed and studied for capillary bridges subject to extension $[39,40,43]$. However, the definition of the critical equilibrium [39] for a pendant bridge implies that during extension the first and second derivatives of total energy of the system with respect to the variable extension of the bridge, both reach zero. In the evaporation case, the bridge extension is imposed as constant, and the driving force is the variable (evaporating) volume, so the definition needs to be reformulated. Padday et al. [39] also observed the liquid wire formation, and its terminal pinching in a no-gravity experiment with a floating-endplate pendant bridge. Like in our experiments, a symmetric pinching of the liquid wire takes place at the extremities, where the wire transitions into conical liquid bodies occurs. Neither Padday et al. [39] nor Peregrine et al. [40] considered the Laplace pressure evolution.

In addition, most of the shape transformations observed during our evaporation experiments were also seen in experiments during steady 
liquid jet gravity flow as early as 1864 by Plateau [38], that is for a completely different flow velocity regime and boundary conditions.

The common thread for this collection of different conditions seems to be an ability to sustain spatially constant Laplace pressure, while the effects like contact (or triple) line pinning, depinning or re-pinning, seem to prompt instable transitions between such alternative states.

The main question yet to be answered is about what causes the observed complex evolution of contact line diameter, including its initial pinning, depinning, slowing down, and eventual re-pinning. Similar questions pertain to the variability of contact angle. It may very well be that the causes are purely local (bead surface roughness, liquid contamination, as widely considered in sessile drop evaporation studies (since Wenzel [44], Cassie and Baxter [45] and Bormashenko et al. [46]), but may likely be global equilibrium instability, bridge external surface or energy minimization constrained additionally by the anchoring effects of the triple line or by triple line tension evolution, in analogy to Cassie-Baxter state instability in sessile drops (Bormashenko and Whyman [47]) (the latter has been suggested by an anonymous Reviewer of the paper). Surface roughness and/contamination may safely be considered as random, while the events of pinning, depinning and re-pinning appear to be repeatable.

Notably, while for evaporation the above-described first (suction) instability, linked to the $C D$ re-pinning occurs shortly before the other two mentioned instabilities, of wire formation, and wire pinching instability take place. But for extension, it is different. In terms of change in separation, suction instability occurs way before the wire formation, and wire rupture.

Finally, it should be noticed that the appearance of the suction instability, does not imply that other forms, i.e. liquid-wire formation and its final pinching would follow. Indeed for both evaporation and extension processes, the liquid-wire instability is absent for very small separations and small liquid volumes.

\section{Conclusions}

The following highlights summarize the findings of the discussed experiments focused on Laplace pressure evolution during free (constant ambient temperature, relative humidity and air pressure) evaporation of liquid bridges between two glass spheres investigated at different constant grain separations and the same initial liquid volumes:

1. This is the first time to our knowledge that Laplace pressure is calculated from the measured bridge curvatures along the process of evaporation.

2. Laplace pressure (or suction) $\Delta p$ evolves quite significantly and may reach during evaporation the maximum values several times to up to 100 times higher, than the initial value.

3. The Laplace pressure evolution strongly depends on the grain separation and falls into three distinct categories depending on bridge height: tall, short and very short.

4. Except for large separations (tall bridges), for which $\Delta p$ is all the time positive, usually the initial $\Delta p$ is negative (suction) reaching a maximum after more than half of water is evaporated, to subsequently rapidly fall to zero and into positive values, to eventually rupture.

5. Rupture in all cases occurs at a positive Laplace pressure.

6. A bridge at rupture may still contain a large fraction of the initial water volume (especially for tall bridges, i.e. at high separations).

7. In no case did suction, nor attractive intergranular force reach high values reported previously to develop during desaturation in "granular continua".

8. Up to four consecutive forms of instability may develop during evaporation, in the following order: suction instability, suction to pressure transition (or Raileigh nodoid/catenoid instability), unduloid to cylinder (water-wire) transition and pinching of the wire (terminal). The latter two instabilities were not recorded for VSVW bridges. The latter bridges rupture in the middle by evaporation of their central section.

9. Contact angle (apparent) CA varies highly during evaporation, decreasing for tall and short bridges, increasing for very short (VSVW) bridges, mostly exhibiting stick-slip pattern.

10. Contact diameter $C D$ exhibits episod of pinning, depinning and re-pinning (sometimes stick and slip patterns).

11. A consistent correlation between terminal re-pinning event and onset of suction instability has been established, also visible in the analogous extension tests

12. The pinning effects highly resemble those developing during the extension tests and during evaporation or motion of sessile drops.

13. The sequence of evaporating bridge shape transition is observed: a nodoid $(\Delta p<0)$ into a catenoid* $(\Delta p=0)$ into an unduloid $(\Delta p>0)$ into a cylinder (water wire) into a pinched cylinder into rupture; $\left.{ }^{*}\right)$ - actually not observable with the technique used- probably of a too-low time resolution.

14. Such sequence bears similarity to analogous developments during steady fluid jet flows (Plateau sequence), pendant drop and two-grain and grain-plate bridge extension.

The above results have certain puzzling consequences to our understanding of the mechanics of unsaturated granular media. It is widely believed [18-21] that a very high suction develops in granular media in the terminal stages of their desaturation. Our results indicate that at the microscale, suction within capillary bridges between two grains during drying drops to zero and becomes positive in terminal stage before rupturing. The highest value of suction measured in these experiments reached about $10 \mathrm{kPa}$. Whatever happens to suction in capillary bridges, their effect on the actual capillary force is imperceptible because in the final stages of evaporation the cross section area on which this capillary pressure acts, tends to become extremely small, producing at the end an insignificant effect for the inter-granular force. While the behavior of isolated two-sphere liquid bridges may not necessarily be generalized to the behavior of a granular medium, it nevertheless excludes certain behavior locally. Two-grain liquid bridges represent a last stadium of the evolution of evaporating multi-grain bridges.

\section{Acknowledgement}

The authors acknowledge the partial support of US NSF grant \#0700294 of CMMI Division, Geomechanics and Geomaterials Program (T. Hueckel) and support of IRSN, CNRS, MIST laboratory and the Languedoc-Roussillon Regional Government (B. Mielniczuk, M. S. El Youssoufi, T. Hueckel).

\section{References}

[1] B. Mielniczuk, T. Hueckel, M.S. El Youssoufi, Evaporation-induced evolution of the capillary force between two grains, Granul. Matter 16 (2014) 815-828, http://dx doi.org/10.1007/s10035-014-0512-6.

[2] B. Mielniczuk, M.S. El Youssoufi, L. Sabatier, T. Hueckel, Rupture of a liquid bridge between two grains due to its evaporation, Acta Geophys. 62 (2014) 1087-1108, http://dx.doi.org/10.2478/s11600-014-0225-6.

[3] K. Hotta, K. Takeda, K. Linoya, The capillary binding force of a liquid bridge, Powder Technol. 10 (1974) 231-242.

[4] H.M. Princen, Comments on "The effect of capillary liquid on the force of adhesion between spherical solid particles", J. Colloid Interface Sci. 26 (1968) 249-253.

[5] M.J. Adams, S.A. Johnson, J.P.K. Seville, C.D. Willett, Mapping the influence of gravity on pendular liquid bridges between rigid spheres, Langmuir 18 (2002) 6180-6184

[6] W.B. Haines, Studies of the physical properties of soils. II. A note on the cohesion developed by capillarity forces in an ideal soil, J. Agric. Sci. 15 (1925) 529-535.

[7] R.A. Fisher, On the capillarity forces in an ideal soil; correction of formulae given by W. B. Haines, J. Agric. Sci. 16 (1926) 492-505.

[8] H.-J. Butt, M. Kappl, Normal capillary forces, Adv. Colloid Interface Sci. 146 (2009) 48-60.

[9] Ch.D. Willett, M.J. Adams, S.A. Johnson, J.P.K. Seville, Capillary bridges between two spherical bodies, Langmuir 16 (2000) 9396-9405 
[10] J.J. Oertli, The stability of water under tension in the xylem, Z. Pflanzenphysiol. 65 (1971) 195-209.

[11] F. Soulié, F. Cherblanc, M.S. El Youssoufi, C. Saix, Influence of liquid bridges on the mechanical behaviour of polydisperse granular materials, Int. J. Numer. Anal. Methods Geomech. 30 (2006) 213-228.

[12] J.-P. Gras, J.-Y. Delenne, M.S. El Youssoufi, Study of capillary interaction between two grains: a new experimental device with suction control, Granul. Matter 15 (2013) 49-56.

[13] A.W. Adamson, Physical chemistry of surfaces, 3 ed. Wiley, New York and London, 1976.

[14] L.L. Bircumshaw, The surface tension of mixtures of alcohol and water at $25^{\circ}$, Chem. Soc. Trans. 121 (1922) 887.

[15] S. Das, Effect of impurities in the description of surface nanobubbles: Role of nonidealities in the surface layer, Phys. Rev. E 83 (2011) 066315.

[16] S. Pietruszczak, Z. Mroz, Finite-element analysis of deformation of strain-softening materials, Int. J. Numer. Methods Eng. 17 (1981) 327-334.

[17] J.L. Chaboche, Continuum damage mechanics. 1. General concepts, J. Appl. Mech. Trans. ASME 55 (1986) 59-64.

[18] J. Krahn, D.G. Fredlund, On total, matric and osmotic suction, Soil Sci. 114 (1927) 339-348.

[19] I.S. McQueen, R.F. Miller, Calibration and evaluation of a wide-range gravimetric method for measuring moisture stress, Soil Sci. 106 (1968) 225-231.

[20] D.R. Snethen, L.D. Johnson, Evaluation of soil suction from filter paper, Misc. paper gl-80-4, Geotechnical Laboratory, U. S. Army Engineer Waterways Experiment Station, 1968.

[21] H. Peron, T. Hueckel, L. Laloui, An improved volume measurement for determining soil water retention curves, Geotech. Test. J. 30 (2007) 1-8.

[22] A.V. Luikov, Heat and mass transfer in capillary-porous bodies, Pergamon Press, Oxford, UK, 1966.

[23] W.J. Stroud, J.E. Curry, J.H. Cushman, Capillary condensation and snap-off in nanoscale contacts, Langmuir 17 (2001) 688-698.

[24] C. Delaunay, Sur la surface de révolution dont la courbure moyenne est constante, suvi d'une Note de Sturm, J. Math. Pures Appl. 6 (1841) 309-320.

[25] R.D. Deegan, O. Bakajin, T.F. Dupont, G. Huber, S.R. Nagel, T.A. Witten, Capillary flow as the cause of ring stains from dried liquid drops, Nature 389 (1997) 827-829.

[26] J. Kroeger, H.J. Rath, Velocity and elongation rate distributions in stretched polymeric and Newtonian liquid bridges, J. Non-Newtonian Fluid Mech. 57 (1995) 137-153.

[27] C. Bourgès-Monnier, M.E.R. Shanahan, Influence of evaporation on contact angle, Langmuir 11 (1995) 2820-2829.

[28] P.G. de Gennes, Wetting: statics and dynamics, Rev. Mod. Phys. 57 (1985) 827-863.

[29] P.G. de Gennes, F. Brochard-Wyart, D. Quéré, Gouttes, bulles, perles et ondes, Belin, 2002.
[30] T. Hueckel, B. Mielniczuk, M.S. El Youssoufi, Micro-scale study of rupture in desiccating granular media, Proceedings of Geo-Congress 2013, Geotechnical Special Publication GSP, 231, 2013.

[31] T. Hueckel, B. Mielniczuk, M.S. El Youssoufi, L.B. Hu, L. Laloui, A three-scale cracking criterion for drying soils, Acta Geophys. 62 (2014) 1049-1059, http://dx.doi.org/10. 2478/s11600-014-0214-9.

[32] A. Marmur, Soft contact: measurement and interpretation of contact angles, Soft Matter 2 (2006) 12-17.

[33] E. Bormashenko, Wetting of real solid surfaces: new glance on well- known problems, Colloid Polym. Sci. 291 (2013) 339-342, http://dx.doi.org/10.1007/s00396012-2778-8.

[34] L.D. Landau, E.M. Lifshitz, Course of Theoretical Physics vol. 6, Fluid mechanics, Pergamon Press, Oxford, 1959.

[35] S. Brandon, A. Wachs, A. Marmur, Simulated contact angle hysteresis of a threedimensional drop on a chemically heterogeneous surface: A numerical example, J. Colloid Interface Sci. 191 (1997) 110-116.

[36] M.E.R. Shanahan, Simple theory of stick-slip wetting hysteresis, Langmuir 11 (1995) 1041-1043.

[37] G. Gagneux, O. Millet, Analytic calculation of capillary bridge properties deduced as an inverse problem from experimental data, Transp. Porous Media 105 (2014) 117-139.

[38] J. Plateau, The figures of equilibrium of a liquid mass, The Annual Report of the Smithsonian Institution, Washington DC 1864, pp. 338-369.

[39] J.F. Padday, G. Pétré, C.G. Rusu, J. Gamero, G. Wozniak, The shape, stability and breakage of pendant liquid bridges, J. Fluid Mech. 352 (1997) 177-204, http://dx. doi.org/10.1017/S0022112097007234.

[40] D.H. Peregrine, G. Shoker, A. Symon, The bifurcation of liquid bridges, J. Fluid Mech. 212 (1990) 25-39, http://dx.doi.org/10.1017/S0022112090001835.

[41] B.L. Weeks, M.W. Vaughn, J.J. DeYoreo, Direct imaging of meniscus formation in atomic force microscopy using environmental scanning electron microscopy, Langmuir 21 (2005) 8096-8098.

[42] D. Vaynblat, J.R. Lister, T.P. Witelski, Symmetry and self-similarity in rupture and pinchoff: a geometric bifurcation, Eur. J. Appl. Math. 12 (2001) 209-232.

[43] M.A. Erle, D.C. Dyson, N.R. Morrow, Liquid bridges between cylinders, in a torus, and between spheres, AICHE J. 17 (1971) 115-121.

[44] R.N. Wenzel, Resistance of solid surfaces to wetting by water, Ind. Eng. Chem. 28 (1936) 988-994.

[45] A.B.D. Cassie, S. Baxter, Wettability of porous surfaces, Trans. Faraday Soc. 40 (1944) 546-551.

[46] E. Bormashenko, E.A. Musin, G. Whyman, M. Zinigrad, Wetting transitions and depinning of the triple line, Langmuir 28 (2012) 3460-3464.

[47] E. Bormashenko, G. Whyman, On the role of the line tension in the stability of Cassie wetting, Langmuir 29 (2013) 5515-5519, http://dx.doi.org/10.1021/la400625e. 